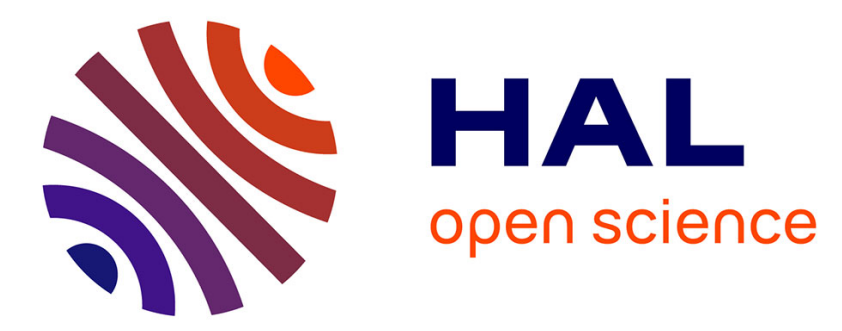

\title{
Three-dimensional flow in fractured porous media: A potential solution based on singular integral equations
} Ahmad Pouya

\section{To cite this version:}

Ahmad Pouya. Three-dimensional flow in fractured porous media: A potential solution based on singular integral equations. Advances in Water Resources, 2011, 35, pp.30-40. 10.1016/j.advwatres.2011.10.009 . hal-00668190

\section{HAL Id: hal-00668190 https://hal.science/hal-00668190}

Submitted on 9 Feb 2012

HAL is a multi-disciplinary open access archive for the deposit and dissemination of scientific research documents, whether they are published or not. The documents may come from teaching and research institutions in France or abroad, or from public or private research centers.
L'archive ouverte pluridisciplinaire HAL, est destinée au dépôt et à la diffusion de documents scientifiques de niveau recherche, publiés ou non, émanant des établissements d'enseignement et de recherche français ou étrangers, des laboratoires publics ou privés. 


\title{
Three-dimensional flow in fractured porous media: a potential solution based on singular integral equations
}

\author{
Ahmad Pouya \\ Université Paris-Est, Laboratoire Navier (UMR CNRS-IFSTTAR-ENPC), \\ IFSTTAR, 58 bd Lefebvre, 75732 Paris, France \\ ahmad.pouya@enpc.fr
}

\begin{abstract}
Governing equations for flow in three-dimensional heterogeneous and anisotropic porous media containing fractures or cracks with infinite transverse permeability are described. Fractures are modeled as zero thickness curve surfaces with the possibility of multiple intersections. It is assumed that flow obeys to an anisotropic Darcy's law in the porous matrix and to a Poiseuille type law in fractures. The mass exchange relations at fractures intersections are carefully investigated as to establish a complete mathematical formulation for the flow problem in a fractured porous body. A general potential solution, based on singular integral equations, is established for steady state flow in an infinite fractured body with uniform and isotropic matrix permeability. The main unknown variable in the equations is the pressure field on the crack surfaces, reducing thus from three to two the dimension of the numerical problem. A general transformation lemma is then given that allows extending the solution to matrices with anisotropic permeability. The results lead to a simple and efficient numerical method for modeling flow in three-dimensional fractured porous bodies.
\end{abstract}

Keywords: porous material, fracture, crack, steady state flow, singular integral equations, boundary element method

\section{Introduction}

Modelling the flow in fractured porous media has a great interest for applications to various industrial problems such as petroleum reservoirs engineering, radioactive waste disposal, $\mathrm{CO}_{2}$ geological storage, water resources management etc. Different aspects of flow in fractured porous media, especially in the contexts of petroleum reservoirs, have been investigated by different numerical methods such as Finite Elements [1, 2, 3], Finite Volume [4, 5, 6] and Discrete Fracture Network [7,8] among many others. In many problems related to flow in fractured geological formations or in micro-cracked porous rocks, one is interested in the steady state flow that takes place under given farfield conditions. This occurs especially when the effective permeability of fractured reservoirs or of microcracked rocks is investigated [4, $9,10,11,12]$. For this purpose, the flow has to be determined in and around the cracks embedded in an infinite matrix with uniform permeability. To model steady state flow in an infinite fractured matrix, Singular Integral Equations provide a powerful method that allows developing simple numerical models, general potential solutions and, in some cases, analytical solutions. 
In potential solutions, the pressure field in the whole body is built as a function of the infiltration in the fractures. This reduces the dimension of the unknown variable field from three (pressure field) to two (infiltration on crack surfaces) and thus simplifies numerical modelling. For 2D plane flow in cracked bodies, potential solutions based on singular integral equations was introduced first by Liolios \& Exadaktylos [13], by using complex number variables. Their solution was restricted to isotropic matrix permeability and, also, excluded crack intersections. The cracks intersections constitute, in fact, a difficulty that mathematical formulations and numerical methods have to handle. A direct formulation of two-dimensional flow in terms of singular integral equations allowed Pouya \& Ghabezloo [14] extending the previous solutions to anisotropic matrices. Furthermore, the integration of mass balance relation at crack intersections allowed them to extend their solution to intersecting cracks. The initial objective of the present work is the extension of these results to three-dimensional flow. However, we realized that this extension faces the problem of mass balance conditions on fractures intersection lines that has not been sufficiently well studied in the literature.

As a matter of fact, in presence of fractures, the formulation of the flow problem has to integrate mass balance conditions concerning matrix-fracture exchanges along fracture surfaces as well as matrix-fracture and fracture-fracture exchanges along fracture intersection lines. The relations governing matrix-fracture exchanges along fracture surfaces have been first expressed by Barenblatt et al. [15] in a context of double porosity concept, and then widely used in subsequent work $[4,6,16,17]$. Their extension to curved fracture surfaces, however, should be examined carefully as it will be shown in this paper. But a review of different numerical modelling works shows that, at least in the context of three-dimensional flow, mass balance conditions at fracture intersections have not been explicitly and clearly formulated. Probably, the concept of double porosity in which the flow in the fracture network and in the matrix are described separately by a doublet of governing equations has made the problem more complicated [18]. It can be noticed that in many works fractures intersections have been simply excluded to avoid conceptual and numerical difficulties. In some works, dealing with $2 \mathrm{D}$ flow, the mass balance at fracture intersection points is expressed only in the numerical model: Granet et al. [5] impose some relations on nodal velocities in elements adjacent to an intersection point in order to assure the mass balance. But concerning the 3D intersecting fractures, we could not find any expression of mass balance conditions on intersection lines and points. However, whatever the numerical method used, a rigorous and complete mathematical formulation of the flow problem integrating these conditions is a prerequisite to establishment of relevant numerical models.

In this work, the fractures represent physically the limits of vanishingly thin fractures or very permeable layers in which the fluid is in equilibrium with the pores fluid, and so, the pressure is continuous at the matrix-fracture interface. Also, there is no pressure jump between the two opposite sides of the fracture, corresponding to an assumption of infinite transverse permeability for the fracture. These assumptions exclude the cases of non-equilibrium flow with different pressures in the fracture and the matrix [19] or of fractures, such as some shear faults, acting as impermeable membranes [20]. Geometrically, the fractures are represented by curved surfaces that may have multiple intersections along different lines and points. In this framework, the paper focuses on the mathematical formulation of the flow problem. First the mass balance equations at intersection lines and points are investigated. Then, a potential solution for the steady state flow is established in an infinite fractured body with uniform matrix permeability. The potential solution allows reducing the $3 \mathrm{D}$ problem to a $2 \mathrm{D}$ problem involving only the flow on fracture surfaces and so making it possible to solve the problem by using 2D numerical methods. The equations for 2D problem are written by supposing a Poiseuille type law (linear relation between infiltration and pressure gradient) for the tangent 
flow in the fracture, but they can be easily extended to non linear laws. The potential solution is first established for isotropic matrix permeability and then extended to anisotropic permeability by using a linear transformation method that will be introduced beforehand.

\section{Notations :}

In the sequel, light-face (Greek or Latin) letters denote scalars; underlined letters denote vectors, bold-face letters designate second rank tensors or double-index matrices. The scalar product of two vectors $\underline{a}$ and $\underline{b}$ is labelled as $\underline{a} . \underline{b}$. For second rank tensors, the tensor transposed from $\boldsymbol{A}$ is denoted $\boldsymbol{A}^{\mathrm{T}}$, the matrix product is labelled as $\boldsymbol{A} \boldsymbol{B}$ and the determinant as $|\boldsymbol{A}|$. The operation of $\boldsymbol{A}$ on $\underline{a}$ is labelled as $\boldsymbol{A} . \underline{a}$.

The Greek indices $(\alpha, \beta \ldots)$ take the values $\{1,2\}$, and the convention of summation on repeated indices is used implicitly for them. This convention is not used for Latin indices $(i, j$, $k \ldots$... that are used to number surfaces, lines, etc., and are noted indifferently as subscript or upperscript. $\nabla$ represents the gradient and $\Delta$ the Laplace operator for a scalar field and $(\nabla$. the divergence for a vector field.

\section{Basic equations}

A three dimensional heterogeneous porous body $\Omega$ containing a set of fracture surfaces is considered. The fractures are numbered by $j$ and denoted $\Gamma_{j}$. The surface $\Gamma_{j}$ is defined by a smooth function $\underline{z}^{j}(\underline{s})$ from $\mathbb{R}^{2} \rightarrow \mathbb{R}^{3}$, where $\underline{s}=\left(s_{1}, s_{2}\right)$ represents the curvilinear parameters. At its boundary, denoted by $\partial \Gamma_{j}$, the crack $\Gamma_{j}$ can end in the matrix, intersect other cracks or outcrop on the boundary of $\Omega$, denoted by $\partial \Omega$. Two or more cracks can intersect along an intersection line and several intersection lines can intersect at an intersection point (Figure 5). The intersection line number $i$ is designated by $L_{i}$. The notation $\Gamma$ or $L$ is used for an arbitrary crack surface or intersection line. Also, without any risk of confusion, $\Gamma$ and $L$ designate the set of all points located respectively on crack surfaces and intersection lines. Thus, we have $L \subset \Gamma \subset \Omega$. The set $\Omega-\Gamma$ is called matrix points, $\Gamma-L$, the regular points on crack surfaces and $L$, singular points.

The matrix and fractures are supposed to be saturated and the flow monophasic. The case of fractures that are the limits of vanishingly thin, empty or very permeable layers, are taken into consideration. There is no pressure jump between two opposite sides of the fracture. The pressure in the fracture is the same that on its interface with the matrix. The flow is characterised by a pressure field $p(\underline{x})$, a velocity field $\underline{v}(\underline{x})$ in the matrix, and an infiltration filed $\underline{q}(\underline{s})$ in the fractures. The normal component of $\underline{v}$ can be discontinuous from one side of the fracture surface to the other, whereas its tangential component remains continuous because of the continuity of the pressure. The infiltration $q$ is defined as the integral of the

fluid velocity $\underline{v}$ in the thickness of the physical fracture or layer: $\underline{q}=\int_{0}^{e} \underline{v}(y) d y$ where $e$ represents the thickness of the fracture in the physical model. The vector field $\underline{q}$ is tangent at every point to the crack surface. The fluid mass per unit matrix volume is denoted by $\rho$. When $\rho$ is constant or function of the pressure, there is no jump for its values between two sides of the fracture. Although in the geometric model the fractures have a zero thickness, 
their physical thickness is not zero and they can contain some fluid mass quantity. The fluid mass per unit surface of the fracture is represented by $\eta$. The parameters $\rho$ and $\eta$ can vary if the fluid is supposed (slightly) compressible. Sinks or source points are excluded for seek of simplicity.

The general equations governing the flow in $\Omega$ encompass the mass balance equations, the boundary conditions on different parts of $\partial \Omega$ and the constitutive laws for flow in the matrix and the fractures. They are examined in the following.

\subsection{Mass balance equations}

The local mass balance condition takes different forms depending whether the point is located in the matrix, on crack surfaces or on intersection lines and points. At a matrix point $\underline{x}$ the mass balance reads:

$$
\forall \underline{x} \in \Omega-\Gamma ; \quad \partial \rho / \partial \mathrm{t}+\nabla \cdot[\rho \underline{v}(\underline{x})]=0
$$

where $\rho$ represents the fluid mass per unit volume of the matrix. It is well known that this local condition can be obtained from an integral condition over a volume $V$ surrounding the point $\underline{x}$ : the variation of the fluid mass $\mathcal{M}$ in $V$ must balance the flux $Q$ leaving the boundary $\partial V$ :

with:

$$
\frac{\partial \mathcal{M}}{\partial t}+Q=0
$$

$$
\mathcal{M}=\int_{V} \rho d \omega, \quad Q=\int_{\partial V} \rho \underline{v} \cdot \underline{n} d s
$$

where $\underline{n}$ represents the unit outward normal on the boundary $\partial V$. The equation (1) is then deduced from (2) by using the divergence theorem for a vector field $\underline{u}$ :

$$
\int_{\partial V} \underline{u} \cdot \underline{n} d s=\int_{V} \nabla \cdot \underline{u} d \omega
$$

We use this lemma as a guideline to obtain mass balance conditions for the points located on crack surfaces. But first, we must give the expression of the divergence theorem for domains including discontinuity surfaces. Let $\underline{u}$ be a vector field through $\Omega$ which is discontinuous across a set of surfaces $\Gamma_{j}$. The two sides of $\Gamma_{j}$ are denoted by $\Gamma_{j}^{-}$and $\Gamma_{j}^{+}$and $\Gamma_{j}$ is oriented by the unit normal $\underline{n}$ pointing from $\Gamma_{j}^{-}$to $\Gamma_{j}^{+}$. The values of $\underline{u}$ on the negative and positive sides of $\Gamma_{j}$ are denoted by $\underline{u}^{-}$and $\underline{u}^{+}$and the jump or discontinuity of $\underline{u}$ through this surface is represented by $\llbracket \underline{u} \rrbracket=\underline{u}^{+}-\underline{u}^{-}$. The extended divergence theorem for $\underline{u}$ in $\Omega$ is expressed by the following mathematical identity:

$$
\int_{V} \nabla \cdot \underline{u} d \omega=\int_{\partial V} \underline{u} \cdot \underline{n} d s-\sum_{j} \int_{\Gamma_{j}} \llbracket \underline{u} \rrbracket \cdot \underline{n} d s
$$

We use this relation to derive the mass balance equations at regular points on crack surfaces and at intersection lines and points. As a matter of fact, the fluid entering the fracture on one side with the velocity $\underline{v}^{-}$, partly joins the tangent flow in the fracture and partly exits the other side with the velocity $\underline{v}^{+}$. The jump $\underline{v}^{+}-\underline{v}^{-}$represents the balance of mass exchanged between matrix and fracture. 


\subsubsection{Mass balance for regular points on the cracks}

For a regular point $\underline{z}$ on a crack $\Gamma_{j}$, it is possible to consider a small volume $V$ surrounding this point and not intersecting any other crack than $\Gamma_{j}$. We note $\Gamma_{j}^{V}$ the portion of $\Gamma_{j}$ bounded by $V$, and by $\gamma_{j}^{V}$ its intersection with $\partial V$ (Figure 1). The total mass $\mathcal{M}$ in $V$ has the following expression:

$$
\mathcal{M}=\int_{V} \rho d \omega+\int_{\Gamma_{j}^{V}} \eta d s
$$

The total flux $Q$ leaving $V$ is the sum of flux passing through the porous surface $\partial V$ and the infiltration leaving the crack on the line $\gamma_{j}^{V}$ :

$$
Q=\int_{\partial V} \rho \underline{v} \cdot \underline{n} d s+\int_{\gamma_{j}^{V}} \underline{q} \cdot \underline{m} d l
$$

In the last integral, $\underline{q}$ represents the infiltration vector on $\Gamma_{j}$ and $\underline{m}$ is the third vector of the Darboux frame, or the unit tangent normal, of $\gamma_{j}^{V}$ on $\Gamma_{j}$ : it is tangent to the surface $\Gamma_{j}$ and normal to the line $\gamma_{j}^{V}$ (see Appendix A). By using the divergence theorem, the first integral is transformed to:

$$
\int_{\partial V} \rho \underline{v} \cdot \underline{n} d s=\int_{V} \nabla \cdot(\rho \underline{v}) d \omega+\int_{\Gamma_{j}} \rho \llbracket \underline{v} \rrbracket \cdot \underline{n} d s
$$

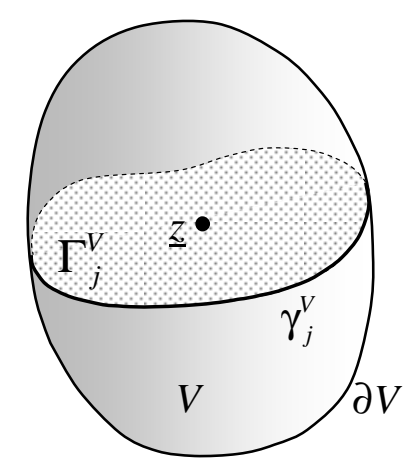

Figure 1 : Small volume $V$ surrounding a regular point $\underline{z}$ on a crack surface $\Gamma_{j}$

The second integral in (7) can be transformed by using the divergence theorem for surfaces.

$$
\int_{\gamma_{j}^{V}} \underline{u} \cdot \underline{m} d l=\int_{\Gamma_{j}} \nabla_{s} \cdot \underline{u} d s
$$

where $\nabla_{s} \cdot \underline{u}$ represents the surface divergence of the field $\underline{u}$. For the surfaces defined by the smooth function $\underline{z}(\underline{s})$, the two vectors $\underline{t}_{\alpha}=\partial \underline{z} / \partial \mathrm{s}_{\alpha}(\alpha=1,2)$ define the natural frame on $\Gamma_{j}$. The contravariant coordinates $u^{\alpha}(\alpha=1,2)$ of a tangent vector field $\underline{u}$ in the frame $\left\{\underline{t} \underline{t}^{\alpha}\right\}$ are defined by the relation $\underline{u}=u^{\alpha} \underline{t}_{\alpha}$ (implicit summation on $\alpha$ ). For plane surfaces, the natural frame remains constant through the surface and the surface divergence of $\underline{u}$ is then given by the following expression:

$$
\nabla_{s} \cdot \underline{u}=\partial_{\alpha} u^{\alpha}
$$

But for curved surfaces, the frame $\left\{\underline{t}^{\alpha}\right\}$ varies on $\Gamma$ and $\nabla_{s} \cdot \underline{u}$ expression has to be replaced by covariant derivative as follows: 


$$
\nabla_{s} \cdot \underline{u}=\partial_{\alpha} u^{\alpha}+u^{\beta} C_{\alpha \beta}^{\alpha}
$$

The coefficients $C_{\alpha \beta}^{\gamma}$ are the Christoffel symbols of the surface $\Gamma$ representing its curvature (see Appendix A). For curved surfaces, the theorem (9) holds only if the true expression (11) for $\nabla_{s} . \underline{u}$ is used.

Combination of (9), (8), (7) and (6) provides:

$$
\frac{\partial \mathcal{M}}{\partial t}+Q=\int_{V}\left(\frac{\partial \rho}{\partial t}+\nabla \cdot(\rho \underline{v})\right) d s+\int_{\Gamma_{j}^{V}}\left(\frac{\partial \eta}{\partial t}+\rho \llbracket \underline{v} \rrbracket \cdot \underline{n}+\nabla_{s} \cdot(\rho \underline{q})\right) d s
$$

The equations (1) and (2) then imply that the last integral in (12) has to vanish for every small surface $\Gamma_{j}^{V}$ around $\underline{z}$, and this implies:

$$
\forall \underline{z}(\underline{s}) \in \Gamma-L ; \quad \frac{\partial \eta(\underline{s})}{\partial t}+\rho \llbracket \underline{v}(\underline{z}) \rrbracket \cdot \underline{n}(\underline{s})+\nabla_{s} \cdot[\rho \underline{q}(\underline{s})]=0
$$

This equation has been widely used in the literature $[16,17,15,4,6]$. However it has generally been obtained by considering the mass exchanges around a point $\underline{z}$ in a small domain $D$ in the tangent plane to $\Gamma$ (Figure 2). The mass balance is written for the exchanges between this domain and the matrix via the upper and lower surfaces $D^{+}$and $D^{-}$and with the remaining parts of the of the crack via the boundary $\partial D$. This latter contribution is transformed to the integral of $\nabla_{s} \cdot \underline{q}$ through $D$ by using the surface divergence theorem, and (13) is obtained. But in this way the expression (10) is supposed for $\nabla_{s} \cdot \underline{q}$ since $D$ is a plane surface. This method does not allow taking account of the curvature effects.

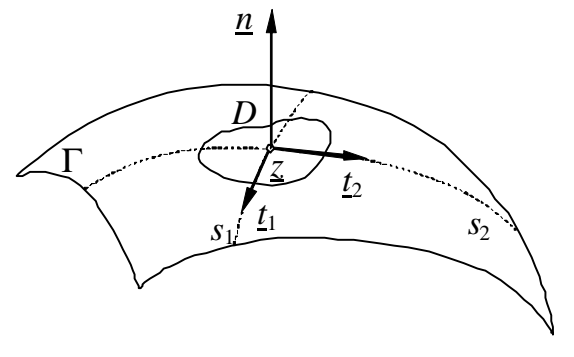

(a)

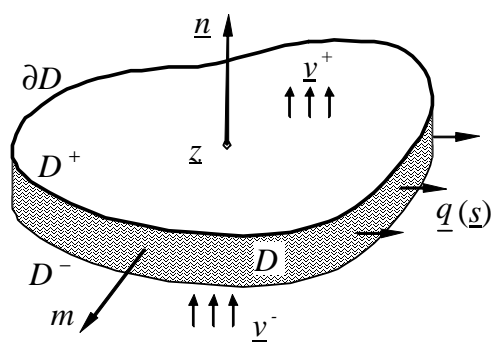

(b)

Figure 2: a) small domain $D$ in the tangent plane to $\Gamma$ around a point $\underline{z}$, b) contributions to the mass exchange for domain $D$

\subsubsection{Mass balance on intersection lines}

Let $L$ be a line along which intersect several cracks $\Gamma_{j}$ and consider a cylindrical shape volume around this line (Figure 3a). The lateral surface of this volume is denoted by $P$, and its two end sections by $S^{+}$and $S^{-}$. The line $L$ is oriented from $S^{-}$to $S^{+}$. If there are $N$ intersecting cracks on $L$, their intersections with $S^{+}, S^{-}$and $P$ divide each one of these surfaces into $N$ sectors. We denote by $P_{j, j+1}, S_{j, j+1}^{+}$and $S_{j, j+1}^{-}$the sectors comprised between the cracks $\Gamma_{j}$ and $\Gamma_{j+1}$ (with the convention $\Gamma_{N+1}=\Gamma_{1}$ to have a cyclic notation). The portion of the crack $\Gamma_{j}$ within the volume $V$ is denoted by $\Gamma_{j}^{V}$. It is delimited by four lines that are a portion of $L$ denoted by $\gamma_{L}$ and the intersections of $\Gamma_{j}$ with the surfaces $P, S^{+}$and $S^{-}$denoted 
respectively by $\gamma_{j}^{P}, \gamma_{j}^{S+}$ and $\gamma_{j}^{S-}$. The portion of $V$ comprised between $\Gamma_{j}$ and $\Gamma_{j+1}$ is denoted by $V_{j, j+1}$. It is delimited by five surfaces that are $\Gamma_{j}^{V}, \Gamma_{j+1}^{V}, S_{j, j+1}^{+}, S_{j, j+1}^{-}$and $P_{j, j+1}$.

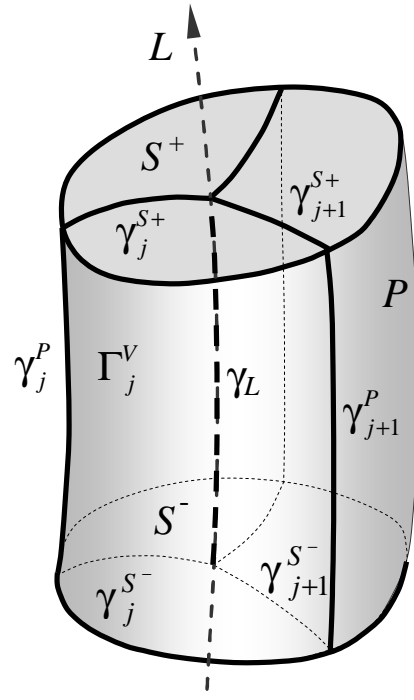

(a)

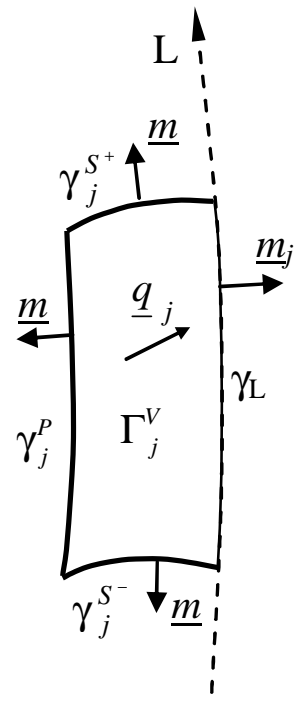

(b)

Figure 3: (a) A small reference volume around the crack intersection line $L$. (b) Portion of the crack surface $\Gamma_{j}$ comprised in the volume and delimited by the lines $\gamma_{j}^{S+}, \gamma_{j}^{S-}, \gamma_{j}^{P}$ and $\gamma_{L}$.

The mass variation in $V$ reads:

$$
\frac{\partial \mathcal{M}}{\partial t}=\sum_{j} \int_{V_{j, j+1}} \frac{\partial \rho}{\partial t} d \omega+\sum_{j} \int_{\Gamma_{j}^{V}} \frac{\partial \eta}{\partial t} d s
$$

The flux of mass leaving this volume reads:

$$
Q=\int_{P} \rho \underline{v} \cdot \underline{n} d s+\int_{S^{+}} \rho \underline{v} \cdot \underline{n} d s+\int_{S^{-}} \rho \underline{v} \cdot \underline{n} d s+\sum_{j} \int_{\gamma_{j}^{p}} \rho \underline{q} \cdot \underline{m} d l+\sum_{j} \int_{\gamma_{j}^{S^{+}}} \rho \underline{q} \cdot \underline{m} d l+\sum_{j} \int_{\gamma_{j}^{S^{-}}} \rho \underline{q} \cdot \underline{m} d l
$$

Let consider now the masse balance (2) in the sub-volume $V_{j, j+1}$ bounded by the surfaces $P_{j, j+1}, S_{j, j+1}^{+}, S_{j, j+1}^{-}, \Gamma_{j}^{V}$ and $\Gamma_{j+1}^{V}$ (Figure 4.a). It leads to the following equality:

$$
\int_{V_{j, j+1}} \frac{\partial \rho}{\partial t} d \omega+\int_{P_{j, j+1}} \rho \underline{v} \cdot \underline{n} d s+\int_{S_{j, j+1}^{+}} \rho \underline{v} \cdot \underline{n} d s+\int_{S_{j, j+1}^{-}} \rho \underline{v} \cdot \underline{n} d s+\int_{\Gamma_{j+1}^{V}} \rho \underline{v}^{-} \cdot \underline{n} d s-\int_{\Gamma_{j}^{V}} \rho \underline{v}^{+} \cdot \underline{n} d s=0
$$

$\underline{n}$ is the unit normal vector on the integration surface. On the surfaces $P, S^{+}$and $S^{-}$, this vector points outward from the volume and, on the surfaces $\Gamma_{j}$, is oriented from the surface $\Gamma_{j}$ to the volume $V_{j, j+1}$. On $\Gamma_{j}$ the fluid velocity is discontinuous: $\underline{v}^{-}$and $\underline{v}^{+}$represent the velocity at respectively the negative and positive sides of this surface orientated by $\underline{n}$ (Figure 4.b). This convention determines the sign of the two last integrals in (16). When summing this equation on $j$, the contribution of the two last integrals can be transformed into expressions involving the jump of $\underline{v}$ on $\Gamma_{j}$. The following result is then found:

$$
\int_{V} \frac{\partial \rho}{\partial t} d \omega+\int_{P} \rho \underline{v} \cdot \underline{n} d s+\int_{S^{+}} \rho \underline{v} \cdot \underline{n} d s+\int_{S^{-}} \rho \underline{v} \cdot \underline{n} d s-\sum_{j} \int_{\Gamma_{j}} \rho \llbracket \underline{v} \rrbracket . \underline{n} d s=0
$$




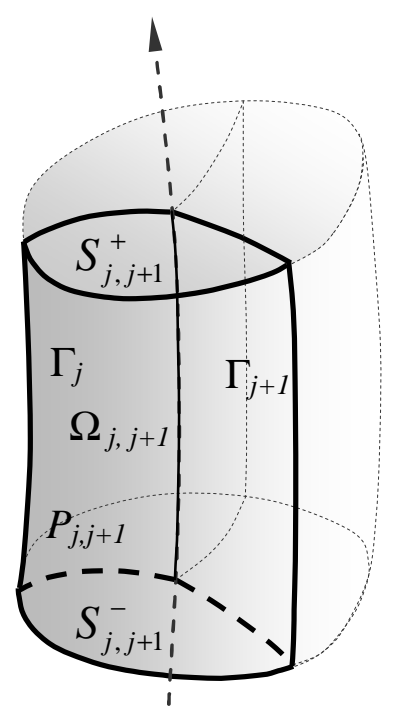

(a)

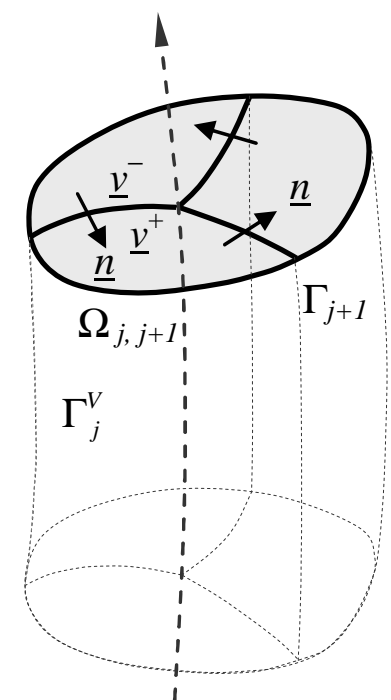

(b)

Figure 4: (a) The sub-volume $V_{j, j+1}$ delimited by the surfaces $P_{j, j+1}, S_{j, j+1}^{+}$, $S_{j, j+1}^{-}, \Gamma_{j}^{V}$ and $\Gamma_{j+1}^{V}$. (b) The orientation of the vector $\underline{n}$ on the surface $\Gamma_{j}$ form this surface to the volume $V_{j, j+1}$

Using (13), the last integral in (17) can be replaced by:

$$
-\sum_{j} \int_{\Gamma_{j}^{V}} \rho \llbracket \underline{v} \rrbracket \cdot \underline{n} d s=\sum_{j} \int_{\Gamma_{j}^{V}} \frac{\partial \eta}{\partial t} d s+\sum_{j} \int_{\Gamma_{j}^{V}} \nabla_{s} \cdot(\rho \underline{q}) d s
$$

The surface divergence theorem for the last integral in (18) reads:

$$
\int_{\Gamma_{j}^{V}} \nabla_{s} \cdot(\rho \underline{q}) d s=\int_{\gamma_{j}^{p}} \rho \underline{q} \cdot \underline{m} d l+\int_{\gamma_{j}^{S+}} \rho \underline{q} \cdot \underline{m} d l+\int_{\gamma_{j}^{S-}} \rho \underline{q} \cdot \underline{m} d l+\int_{\gamma_{L}} \rho \underline{q}_{j} \cdot \underline{m}_{j} d l
$$

In this relation, $\underline{q}$ is the infiltration vector on $\Gamma_{j}$ and $\underline{m}$ on each line is the tangent normal of the line on $\Gamma_{j}$ pointing outward from the domain (Figure 3b). For the last integral, these quantities are specified by the index $j$ because on the line $L$ several cracks meet and specification by $j$ is necessary to distinguish the contribution of different cracks. Then, summing (19) on $j$ and combining it with the relations (18), (17), (15) and (14) results in:

$$
\frac{\partial \mathcal{M}}{\partial t}+Q=-\sum_{j} \int_{\gamma_{L}} \rho \underline{q}_{j} \cdot \underline{m}_{j} d l
$$

To satisfy the mass balance, the left-side of this equation must be null for every portion $\gamma_{L}$. Hence, at every point on $L$ :

$$
\forall \underline{z}(l) \in L ; \quad \quad \sum_{j} \underline{q}_{j} \cdot \underline{m}_{j}=0
$$

Thus, the mass balance on an intersection line of several cracks imposes a condition on the normal components of the infiltration in each crack to the intersection line.

It is worth noting that this relation does not involve exchanges with the matrix and so is independent of the flow in the matrix. This relation is the same as the one would be found for 
a fracture network in an impermeable matrix. This is an important point that has not been clearly emphasized in the literature. It brings significant simplification for mathematical and numerical modelling of flow in fractured porous media.

An important consequence of this relation is that on the boundary of cracks ending in the matrix, the normal component of the infiltration to the boundary line vanishes: $\underline{q} \cdot \underline{m}=0$.

It should be also noted that the intersection lines do not contain a specific fluid mass. It is possible to introduce in the model some singular lines with a specific mass content characterized by a line density. This would be useful to model, for instance, wellbores in an oil reservoir [6]. But this is physically a context different from the fracture intersection lines that are considered here above.

\subsubsection{Mass balance at intersection points}

The existence of intersection points is unavoidable when, for instance, there are three families of mutually orthogonal fractures in a rock formation. At an intersection point, an intersection line intersects other intersection lines and fracture surfaces. The velocity and infiltration fields can present different discontinuities around intersection points, and mass balance equations must include the conditions around these points.

Let the intersection line $L$ considered in the previous section be cut by a new crack surface $\Gamma_{c}$. The cylindrical volume around $L$, as well as the surfaces $P$ and $\Gamma_{j}$ and the lines $\gamma_{j}^{P}$ and $\gamma_{L}$ are cut by $\Gamma_{c}$ in two parts that can be designated, respectively, by $V^{+}$and $V^{-}, \gamma_{j}^{P+}$ and $\gamma_{j}^{P-}$ etc. (Figure 5). In addition, intersection of $\Gamma_{c}$ with $P$ and $\Gamma_{j}$ defines new lines designated by $\gamma_{c}^{P}$ and $\gamma_{j c}$. On the line $\gamma_{j c}$, we denote by $\underline{q}_{j c}^{+}$and $\underline{q}_{j c}^{-}$respectively the discharges in the surfaces $\Gamma_{j}^{+}$and $\Gamma_{j}^{-}$and by $\underline{m}_{j c}^{+}$and $\underline{m}_{j c}^{-}$the normal unit vectors tangent to $\Gamma_{j}$ and pointing outward respectively from $\Gamma_{j}^{+}$and $\Gamma_{j}^{-}$. Since $\Gamma_{j}$ is smooth, we can denote by $\underline{m}_{c j}$ for $\underline{m}_{j c}^{-}=-$ $\underline{m}_{j c}^{+}$(Figure 5). We denote also, on the same line $\gamma_{j c}$, by $\underline{m}_{c j}$ for the unit tangent normal of the line $\gamma_{j c}$ on $\Gamma_{c}$ pointing from this line to the volume $V_{j, j+1}$ (Figure $4 \mathrm{~b}$ ), and by $\underline{q}_{c j}^{-}$and $\underline{q}_{c j}^{+}$respectively for the infiltration vectors in the surface $\Gamma_{c}$ at the negative and positive sides of $\underline{m}_{c j}$.

Compared to the previous case, the balance of discharge leaving the volume must be completed by the contribution of the line $\gamma_{c}^{P}$. The total mass variation $\partial \mathcal{M} / \partial \mathrm{t}$ in $V$ and discharge $Q^{\prime}$ leaving $V$ read respectively:

$$
\frac{\partial \mathcal{M}^{\prime}}{\partial t}=\frac{\partial \mathcal{M}}{\partial t}+\int_{\Gamma_{c}} \frac{\partial \eta}{\partial t} d s, \quad Q^{\prime}=Q+\int_{\gamma_{C}^{P}} \underline{q} \cdot \underline{m} d l
$$

where $\partial \mathcal{M} / \partial \mathrm{t}$ and $Q$ are given by (14) and (15). The analysis carried out on the volume $V$ in the previous section can be applied to each sub-volume $V^{+}$and $V^{-}$. The upper part is limited, at its lower face by the surface $\Gamma_{c}$ and the lower part, at its upper face, by $\Gamma_{c}$, with outward unit vectors oriented in opposite directions. 


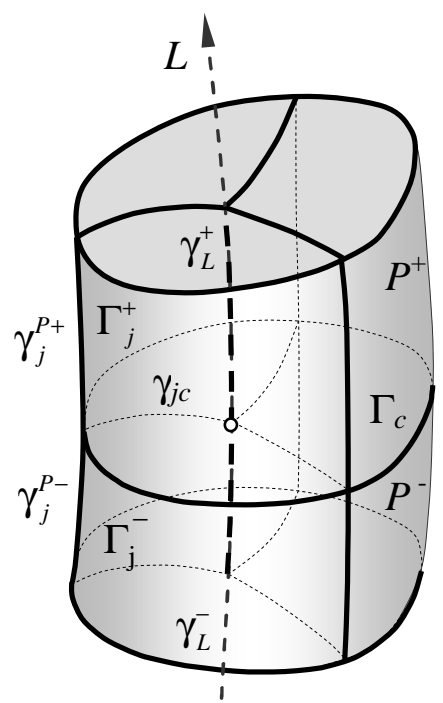

(a)

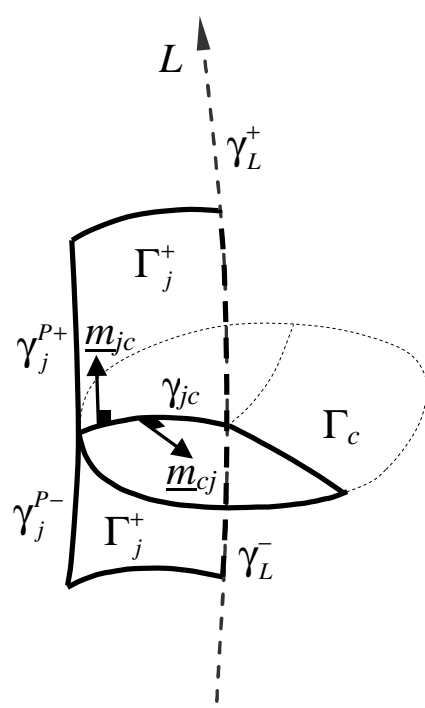

(b)

Figure 5: Small reference volume around an intersection point

Writing the equation (7) for each sub-volume $V^{+}$and $V^{-}$delimited by the surfaces $\Gamma_{j}$ and then summing theses equations for all the sub-domains, an equation analogue to (20) is found as follows:

$$
\left.\frac{\partial \mathcal{M}^{\prime}}{\partial t}+Q^{\prime}=-\sum_{j} \int_{\gamma_{L}^{+}} \underline{q}_{j} \cdot \underline{m}_{j} d l-\sum_{j} \int_{\gamma_{L}^{-}} \underline{q}_{j} \cdot \underline{m}_{j} d l-\sum_{j} \int_{\gamma_{j c}}\left(\underline{q}_{j c}^{-}-\underline{q}_{j c}^{+}\right) \cdot \underline{m}_{j c}+\left(\underline{q}_{c j}^{-}-\underline{q}_{c j}^{+}\right) \cdot \underline{m}_{c j}\right) d l
$$

However, according to the mass balance condition (21), the two first integrals in (14), corresponding to integration on $\gamma_{L}^{+}$and $\gamma_{L}^{-}$, vanish. If the result (21) is applied to the intersection line $\gamma_{j c}$, it implies that:

$$
\left(\underline{q}_{j c}^{-}-\underline{q}_{j c}^{+}\right) \cdot \underline{m}_{j c}+\left(\underline{q}_{c j}^{-}-\underline{q}_{c j}^{+}\right) \cdot \underline{m}_{c j}=0
$$

This means that the condition $\partial \mathcal{M}^{\prime} / \partial \mathrm{t}+Q^{\prime}=0$ already results from (21) without any additional condition. Hence, the mass balance at intersection points does not induce new equations and is included in previous ones. However, it must be noted that the infiltration $\underline{q}$ in a crack surface can be discontinuous when crossing an intersection line, and the mass balance (21) has to take into account this discontinuity. Generally, at intersection points, an intersection line meets a new crack surface. The infiltrations $\underline{q}_{j}$ which appears in (21) takes then different limit values on the two sides of the new crack surface. The condition (21) must be satisfied on each side of the surface. For instance, if we consider the intersection point of the line $L$ with the surface $\Gamma_{c}$ (Figure 5), then $\underline{q}_{j}$ on the lines $\gamma_{L}^{-}$and $\gamma_{L}{ }^{-}$has for limit values respectively $\underline{q}_{j}^{-}$and $\underline{q}_{j}^{+}$when approaching this point. The condition (21) on $\gamma_{L}^{+}$and $\gamma_{L}^{-}$then leads to the following limit conditions at the intersection point:

$$
\sum_{j} \underline{q}_{j}^{-} \cdot \underline{m}_{j}=0, \quad \quad \sum_{j} \underline{q}_{j}^{+} \cdot \underline{m}_{j}=0
$$

In a general way, there are as many equations to be satisfied at an intersection point that there are half-lines departing from this point. These conditions are the limit cases of the condition (21) on each one of these lines at the intersection point. 
In summary, the mass balance for the whole medium is expressed locally by three types of conditions: the condition (1) that involves only the flow in the matrix, the condition (13) governing the matrix-fractures exchanges, and the condition (21) that involves only the flow in fractures and which is the same that for a fractures network in an impermeable matrix.

\subsection{Boundary conditions}

For continuum materials, the boundary $\partial \Omega$ is classically divided into two parts $\partial_{p} \Omega$ and $\partial_{\nu} \Omega$ where respectively the pressure $p$ and the outgoing flux $\underline{v} \cdot \underline{n}$ are imposed. For cracked bodies, a third type of boundary condition must be introduced when a crack outcrops on the boundary. The intersection of a crack surface $\Gamma_{j}$ with $\partial \Omega$ defines a line on this boundary that is designated by $\gamma_{j}^{B}$. On these lines, the pressure or the outflow of the crack have to be specified in order to complete the formulation of the flow problem. When the pressure is prescribed on these lines, it is not necessary to distinguish them from the other parts of $\partial \Omega$ where pressure is imposed. Hence, the notation $\partial_{p} \Omega$ covers all the points on $\partial \Omega$ where the pressure is imposed, including the points located on fractures outcrops. However, it is possible that the fracture outflow is known or imposed on the boundary. This is the case, for instance, when a fluid is injected in a fracture at a constant rate or when the outflow of a fracture outcropping in a tunnel or in a wellbore is measured. The fracture outcrop lines on $\partial \Omega$ where the outflow is imposed are designated by $\partial_{q} \Omega$, and the corresponding outflow by $Q^{g}$. The boundary condition on these lines reads then $\underline{q}_{j} \cdot \underline{m}_{j}=Q^{g}$ with $\underline{q}_{j}$ the infiltration on $\Gamma_{j}$ and $\underline{m}_{\mathrm{j}}$ the tangent normal to $\gamma_{j}^{B}$ on $\Gamma_{j}$ pointing out of $\Omega$. Note that this condition involves the tangent normal $\underline{m}_{j}$ that can be different from the unit normal $\underline{n}$ to $\partial \Omega$.

In summary, the boundary conditions consist in specification of a given pressure $P^{\mathrm{g}}$ on a part $\partial_{p} \Omega$, a normal flux $V^{g}$ outgoing through the matrix on a part $\partial_{v} \Omega$, and a fracture outflow $Q^{\mathrm{g}}$ on a part $\partial_{q} \Omega$ of the boundary.

\subsection{Constitutive laws}

Fluid velocity $\underline{v}(\underline{x})$ in the matrix is given by Darcy's law:

$$
\forall \underline{x} \in \Omega-\Gamma ; \quad \underline{v}(\underline{x})=-k(\underline{x}) . \nabla p(\underline{x})
$$

where $\boldsymbol{k}$ is the local permeability and $p$ is the pressure gradient.

Fluid flow through a planar single crack is a well-known process which is commonly described by Poiseuille's law, at least if roughness effects are negligible. This model is widely used in particular for flow in natural rock joints [21]. It assumes a linear relation between the infiltration $\underline{q}$ and the pressure gradient along the crack surface: $q=-c \nabla p$, where $c$ is called the hydraulic conductivity of the crack. This coefficient is a function of the fluid dynamic 
viscosity $\mu$, hydraulic aperture of the fracture $e$, and also the geometrical properties (roughness) of fracture walls. The assumption of a laminar flow between two infinite and parallel planes leads to the classical cubic law: $c=e^{3} /(12 \mu)$. However, the shape of the asperities on fracture walls can result in different conductivities for different directions of the flow in the fracture. It has been shown experimentally by Gentier et al. [22] that the hydraulic conductivity can be anisotropic in the fracture plane. The anisotropic relationship between the infiltration vector and the in-plane pressure gradient in the fracture is expressed by a tensor $\boldsymbol{c}$ :

$$
\underline{q}(\underline{s})=-c(\underline{s}) \cdot \nabla_{s} p(\underline{s})
$$

The tensor $\boldsymbol{c}$ is symmetric and positive-definite and can vary on the crack surface according to the physical properties of the crack. This type of relation between infiltration and pressure gradient is found also in the case of a thin layer of a material obeying to Darcy's law with a high permeability $\boldsymbol{k}$. The equivalent infiltration $q$ is defined, in this case, as the integral of the fluid velocity $\underline{v}$ in the thickness of the layer: $\underline{q}=\int_{0}^{e} \underline{v}(y) d y$. If the thickness of the layer tends to zero and at the same time its permeability increases in such a way that $e \boldsymbol{k}$ tends to a constant value $c$, then the same type of relation (27) is found between $q$ and $\nabla_{\mathrm{s}} p$ in the limit case.

Since $\nabla_{\mathrm{s}} p$ and $q$ lay both in the tangent plane of the fracture, according to (27), $c$ operates only in this plane and has to be represented by a $2 \times 2$ matrix in this plane. However, it is mathematically easier to represent it as a $3 \times 3$ tensor in the three-dimensional space in which the whole problem is formulated. In this case, the out-of-plane components of this tensor have to be specified. Representing by $\underline{t}_{1}(s)$ and $\underline{t}_{2}(s)$ two orthogonal unit vectors in the tangent plane and by $\underline{n}(s)$ the unit normal to this plane, we must take $\underline{n} \cdot \boldsymbol{c} . \underline{t}_{1}=\underline{n} . \boldsymbol{c} \cdot \underline{t}_{2}=0$ to assure that $q$ has no normal component to the fracture plane. This condition is mathematical necessary because, if for instance in the equation (52) $\underline{x}$ is out of the fracture plane, the results can be affected by the normal component of $q$. Concerning the component $\underline{n} . \boldsymbol{c} . \underline{n}$, the problem is different. This component does not affect any result in the equations and we can give it arbitrary value in the mathematical problem. In numerical methods, it is naturally easier to take $\underline{n} . \boldsymbol{c} . \underline{n}=0$. In this case, the whole condition on the normal components of $\boldsymbol{c}$ can be written as:

$$
c(\underline{s}) \cdot \underline{n}(\underline{s})=0
$$

However, a physical interpretation could be associated to $\underline{n} . \boldsymbol{c} . \underline{n}=0$ that is assuming the transverse permeability of the fracture zone to be zero. This is not conforming to the basic assumption of no pressure jump between the two faces of the fracture which supposes, in contrary, an infinite transverse permeability. To accommodate this possible physical interpretation with the necessary conditions $\underline{n} . \boldsymbol{c} . \underline{t}_{1}=\underline{n} . \boldsymbol{c} . \underline{t}_{2}=0$, it is more suitable to write:

$$
c^{-1}(\underline{s}) \cdot \underline{n}(\underline{s})=0
$$

The difference between (28a) and (28b) resides only in the value of $\underline{n} . \boldsymbol{c} . \underline{n}$. We emphasize that this value has rigorously not any effect in the whole system of equations presented in this paper. The writing (28b) is more conforming to a possible physical interpretations of this value, whereas (28b) is more adapted for numerical calculations. 


\subsection{Mathematical formulation}

The equations to be satisfied by the solution of the flow problem consist in boundary conditions, constitutive relations and mass balance equations. The problem consists in finding the fields $p(\underline{x}), \underline{v}(\underline{x})$ and $\underline{q}(\underline{s})$ through $\Omega$ satisfying the following equations:

$$
\begin{array}{ll}
\text { I.1) } \forall \underline{x} \in \partial_{p} \Omega ; & p(\underline{x})=P^{\mathrm{g}}(\underline{x}), \\
\text { I.2) } \forall \underline{x} \in \partial_{v} \Omega ; & \underline{v}(\underline{x}) \cdot \underline{n}(\underline{x})=V^{\mathrm{g}}(\underline{x}), \\
\text { I.3) } \forall \underline{z}(\underline{s}) \in \partial_{q} \Omega ; & \underline{q}(\underline{s}) \cdot \underline{m}(\underline{s})=Q^{\mathrm{g}}(\underline{z}), \\
\text { I.4) } \forall \underline{x} \in \Omega-\Gamma ; & \underline{v}(\underline{x})=-k(\underline{x}) \cdot \nabla p(\underline{x}), \\
\text { I.5) } \forall \underline{z}(\underline{s}) \in \Gamma ; & \underline{q}(\underline{s})=-c(\underline{s}) \cdot \nabla_{s} p(\underline{z}), \\
\text { I.6) } \forall \underline{x} \in \Omega-\Gamma ; & \partial \rho / \partial \mathrm{t}+\nabla \cdot[\rho \underline{v}(\underline{x})]=0, \\
\text { I.7) } \forall \underline{z}(\underline{s}) \in \Gamma-L ; & \partial \eta / \partial \mathrm{t}+\rho \llbracket \underline{v}(\underline{z}) \rrbracket \cdot \underline{n}(\underline{s})+\nabla_{s} \cdot[\rho \underline{q}(\underline{s})]=0, \\
\text { I.8) } \forall \underline{z}(l) \in L ; & \sum_{j} \underline{q}_{j}(l) \cdot \underline{m}_{j}(l)=0
\end{array}
$$

This set of equations defines completely the mathematical problem to be solved. The last equation constitutes a limit condition for the partial differential equations I.4) to I.7) in the same way that I.1) to I.3) and is as necessary as these equations to complete the mathematical formulation. However, it has not been mentioned in previous works having addressed flow in $3 \mathrm{D}$ fractured porous media.

The existence and uniqueness of the solution to the flow problem can be studied on the basis of these equations. Different numerical methods can be established to solve these equations. In this paper, a potential solution using Singular Integral Equations method will be established for these equations. But, first, we have to introduce a linear transformation method that allows passing from the solution for isotropic matrices to the solution for anisotropic matrices.

\section{Linear transformation}

Methods for transforming complex problems into simpler ones have been frequently developed in various fields of physics. In particular, linear transformations of the coordinates have been used to convert the study of anisotropic bodies into the resolution of problems involving isotropic bodies. This method has been intensively used for the investigation of the hydraulic diffusion in anisotropic porous media [2, 23], for thermal or chemical diffusion [24] and for deformation of elastic bodies [25]. A general transformation method was given by Pouya \& Ghabezloo [14] that allows changing a problem of cracked porous body with anisotropic matrix, to an equivalent problem with isotropic matrix. However this method concerned 2D problems. For 3D problems, the transformation method involves some different relations that will be presented in the following. 
The flow problem in the body $\Omega$ described by the equations I.1 to I.8 is considered. Let $\boldsymbol{M}$ be a reversible tensor and $\zeta$ a positive constant and define the transformed variables $\underline{\tilde{x}}, \underline{\tilde{v}}(\underline{\tilde{x}})$ and $\tilde{p}(\underline{\tilde{x}})$ as follows:

$$
\underline{\tilde{x}}=M \cdot \underline{x}, \quad \underline{\tilde{v}}(\underline{\tilde{x}})=\boldsymbol{M} \cdot \underline{v}(\underline{x}), \quad \tilde{p}(\underline{\tilde{x}})=p(\underline{x}), \quad \tilde{\rho}(\underline{\tilde{x}})=\zeta \rho(\underline{x})
$$

The geometrical transformation $\underline{\tilde{x}}=\boldsymbol{M} . \underline{x}$ changes the domain $\Omega$ to a domain $\tilde{\Omega}$, the cracks $\Gamma$ to $\tilde{\Gamma}$ and the intersection lines $L$ to $\tilde{L}$. At a first step, a variables change in the equations I. 1 to I.8 consists in replacing by (29) for $\underline{x}, \underline{v}$ and $p$ in these equations. Next, a set of adequate variables $\tilde{\rho}, \tilde{\eta}, \tilde{q}, \tilde{\boldsymbol{k}}, \tilde{\boldsymbol{c}}, \tilde{V}^{\mathrm{g}}, \tilde{P}^{\mathrm{g}}$ and $\tilde{Q}^{\mathrm{g}}$ are sought for in such a way that the new equations take formally the same expressions that I.1 to I.8. This means that the new equations have to take the following form:

$$
\begin{aligned}
& \text { II.1) } \forall \underline{\tilde{x}} \in \partial_{p} \tilde{\Omega} ; \quad \tilde{p}(\underline{\tilde{x}})=\tilde{P}^{\mathrm{g}}(\underline{\tilde{x}}), \\
& \text { II.2) } \forall \underline{\tilde{x}} \in \partial_{v} \tilde{\Omega} ; \quad \underline{\tilde{v}}(\underline{\tilde{x}}) \cdot \underline{\tilde{n}}(\underline{\tilde{x}})=\tilde{V}^{\mathrm{g}}(\underline{\tilde{x}}) \text {, } \\
& \text { II.3) } \forall \underline{\tilde{z}}(\underline{\tilde{s}}) \in \partial_{q} \tilde{\Omega} ; \quad \underline{\tilde{q}}(\tilde{s}) \cdot \underline{\tilde{m}}(\underline{\tilde{s}})=\widetilde{Q}^{\mathrm{g}}(\underline{\tilde{z}}) \text {, } \\
& \text { II.4) } \forall \underline{\tilde{x}} \in \tilde{\Omega}-\tilde{\Gamma} ; \quad \underline{\tilde{v}}(\underline{\tilde{x}})=-\tilde{\boldsymbol{k}}(\underline{\tilde{x}}) . \tilde{\nabla} p(\underline{\tilde{x}}) \text {, } \\
& \text { II.5) } \forall \underline{\tilde{z}}(\underline{\tilde{s}}) \in \tilde{\Gamma} ; \quad \underline{\tilde{q}}(\underline{\tilde{s}})=-\tilde{\boldsymbol{c}}(\underline{\tilde{s}}) \cdot \tilde{\nabla}_{s} \tilde{p}(\underline{\tilde{z}}), \\
& \text { II.6) } \forall \underline{\tilde{x}} \in \tilde{\Omega} ; \quad \partial \tilde{\rho} / \partial t+\tilde{\nabla} \cdot[\tilde{\rho} \underline{\tilde{v}}(\underline{\tilde{x}})]=0, \\
& \text { II.7) } \forall \underline{\tilde{z}}(\underline{\tilde{s}}) \in \tilde{\Gamma}-\tilde{L} ; \quad \partial \tilde{\eta} / \partial t+\tilde{\rho} \llbracket \underline{\tilde{v}}(\underline{\tilde{z}}) \rrbracket . \underline{\tilde{n}}(\underline{\tilde{s}})+\tilde{\nabla}_{s} \cdot[\tilde{\rho}(\tilde{s}) \underline{\tilde{q}}(\tilde{s})]=0 \text {, } \\
& \text { II.8) } \forall \underline{\tilde{z}}(\underline{\tilde{l}}) \in \tilde{L} ; \quad \sum_{j} \underline{\tilde{q}}_{j}(\tilde{l}) \cdot \underline{\tilde{m}}_{j}(\tilde{l})=0
\end{aligned}
$$

In these relations we have designated by $\tilde{\nabla}()=.\frac{\partial}{\partial \underline{x}}($.$) .$

The transformation rule for all the variables can be deduced successively from the basic rules (29). For instance, provided that $\nabla p(\underline{x})=\boldsymbol{M}^{T} . \tilde{\nabla} \tilde{p}(\underline{\tilde{x}})$ results from (29), the relation I.4) is transformed to II.4) if we take:

$$
\tilde{\boldsymbol{k}}=\boldsymbol{M} \boldsymbol{k} \boldsymbol{M}^{T}
$$

Determination of the transformation rules for $\underline{\tilde{q}}$ and $\tilde{c}$ is a little more technical. First, note that the unit normal $\underline{\tilde{n}}$ on the surface $\tilde{\Gamma}_{j}$ is related to the unit normal $\underline{n}$ on $\Gamma_{j}$ by the equation $\underline{\tilde{n}}$ $=h_{n} \boldsymbol{M}^{-1, T} \underline{n}$ where (See Appendix A):

$$
h_{n}=\left\|\boldsymbol{M}^{-1, T} \cdot \underline{n}\right\|^{-1}
$$

The transformation rules for $\underline{v}$ and $\underline{n}$ lead to $\llbracket \underline{\tilde{v}} \rrbracket . \underline{\tilde{n}}=h_{n} \llbracket \underline{v} \rrbracket . \underline{n}$, and so, to obtain II.7 from I.7, we must take: 
and have:

$$
\tilde{\eta}=\zeta h_{n} \eta
$$

$$
\tilde{\nabla}_{s} \cdot(\underline{\tilde{\rho}} \underline{\tilde{q}})=\zeta h_{n} \nabla_{s} \cdot(\tilde{\rho} \underline{q})
$$

For plane surfaces, the latter relation will be satisfied if $\tilde{q}^{\alpha}=h_{n} q^{\alpha}$. Taking into account the transformation of the natural frame (see Appendix A), this implies:

$$
\underline{\tilde{q}}=h_{n} \boldsymbol{M} \cdot \underline{q}
$$

It is shown in Appendix B (equation B.3) that for curved surfaces also, the relation (33) implies well (32). However, the question of uniqueness of this solution arises. As a matter of fact, for plane surfaces, (32) implies $\tilde{q}^{\alpha}=h_{n} q^{\alpha}+\psi^{\alpha}$ where $\psi^{\alpha}$ is a pure curl field: $\partial_{\alpha} \psi^{\alpha}=0$. But $\underline{\tilde{q}}$ has to satisfy an additional property that is to be related to a gradient field by a symmetric tensor $\tilde{\boldsymbol{c}}$. It can be shown that, at least for plane surface $\Gamma$ and constant $\boldsymbol{c}$, this condition implies $\psi^{\alpha}=0$ and the solution (33) is, therefore, unique.

The same relation (33) is obtained also by a physical reasoning when the crack is replaced by a thin layer of material. By taking the equivalent infiltration as the integral of the fluid velocity in the layers section, $\underline{q}=\int_{0}^{e} \underline{v} d y$, and applying the transformation rules for $\underline{v}$ and for the thickness $e,(33)$ will be obtained.

Regarding the fractures conductivity, the relation between the surface gradients is $\tilde{\nabla}{ }_{s} \tilde{p}=\boldsymbol{M}^{-1, \mathrm{~T}} \cdot \nabla_{s} p$. Introducing this relation in I.5), II.5) is obtained if:

$$
\tilde{\boldsymbol{c}}=h_{n} \boldsymbol{M} \boldsymbol{c} \boldsymbol{M}^{\mathrm{T}}
$$

To determine the transformation rule for crack boundary conditions, the transformation rule for the tangent normal $\underline{m}$ has to be first determined. The following relation has been shown between initial and transformed quantities in Appendix B:

$$
\underline{\tilde{q}} \cdot \underline{\tilde{m}}=h_{t}|\boldsymbol{M}| \underline{q} \cdot \underline{m}
$$

where $\underline{t}$ is the unit tangent to the crack boundary line and

$$
h_{t}=\|\boldsymbol{M} \cdot \underline{t}\|^{-1}
$$

From the above equations, transformation rules for the boundary conditions derive as follows:

$$
\tilde{P}^{g}(\underline{\tilde{x}})=P^{g}(\underline{x}), \quad \tilde{V}^{g}(\underline{\tilde{x}})=h_{n} V^{g}(\underline{x}), \quad \tilde{Q}^{g}(\underline{\tilde{x}})=\zeta h_{t}|\boldsymbol{M}| Q^{g}(\underline{x})
$$

where $h_{n}$ and $h_{t}$ are related respectively to the outward normal $\underline{n}$ on $\partial \Omega$ and the unit tangent $\underline{t}$ to the line that is the crack trace on $\partial \Omega$.

The definition of the transformed quantities is thus completed. Nevertheless, the coherence of the transformation rules requires examining if the other equations are also satisfied. The remaining equations are the mass balance condition at intersection lines (II.8). Let $L$ be the intersection line between cracks $\Gamma_{j}$. At a point $\underline{x}$ on this line a Darboux frame $\left(\underline{t}, \underline{n}_{j}, \underline{m}_{j}\right)$ can be defined for each crack $\Gamma_{j}$. The vector $\underline{t}$ which is the unit tangent to $L$ is the same for all these frames, and so $h_{t}$ in (35) is the same for all these cracks. This assure that the limit condition II.8) results well from I.8). As a matter of fact, replacing by (35) in I.8) we obtain:

$$
\sum_{j} \underline{\tilde{q}}_{j} \cdot \underline{\tilde{m}}_{j}=h_{t}|\boldsymbol{M}| \sum_{j} \underline{q}_{j} \cdot \underline{m}_{j}=0
$$


The transformed system of equations II.1) to II.8) formally describes the flow in a microcracked porous body with geometry $(\tilde{\Omega}, \tilde{\Gamma})$, permeability $\tilde{\boldsymbol{k}}$, crack conductivity $\tilde{\boldsymbol{c}}$ and boundary conditions $\tilde{V}^{\mathrm{g}}, \tilde{P}^{\mathrm{g}}$ and $\tilde{Q}^{\mathrm{g}}$. Physically, it is a different problem. But the mathematical relations established here-above between the two problems allow deriving the solution of one problem from the solution of the other one. Many partial results of this transformation can be found in the literature. Generally, taking $\boldsymbol{M}=\boldsymbol{\lambda} \boldsymbol{\delta}$ allows obtaining the same results that a dimensional analysis or utilization of dimensionless parameters. The choice of anisotropic $\boldsymbol{M}$ allows obtaining more subtle results. An example of application of this transformation will be seen in Section 5.

\section{A general potential solution for infinite medium}

Determination of the steady state flow in fractured geological formations or in micro-cracked rocks under farfield pressure conditions is the key issue for many theoretical studies and industrial applications. In this section, a potential solution for this problem is established in which the unknown variable is the infiltration field in the cracks. The three-dimensional numerical problem is thus reduced to a two-dimensional one since the numerical resolution will require only discretization of crack surfaces. The potential solution is formulated in terms of singular integral equations. In the following, this solution is first derived for the case of isotropic matrix, and then, by using the transformation rules presented here above, extended to anisotropic matrices.

The body $\Omega$ considered in the following is infinite and homogeneous with an isotropic permeability $k$. The cracks $\Gamma_{j}$ have finite size and so do not intersect the infinite boundary. The body is submitted at its infinite boundary to a pressure field $p_{\infty}(\underline{x})$ :

$$
\lim _{\|x\| \rightarrow \infty}\left[p(\underline{x})-p_{\infty}(\underline{x})\right]=0
$$

$p_{\infty}$ satisfies $\Delta p_{\infty}=0$ where $\Delta$ represents the Laplace operator. In most general cases considered for determination of the equivalent permeability, $p_{\infty}$ corresponds to a uniform pressure gradient: $p_{\infty}(\underline{x})=\underline{A} . \underline{x}$. The equations to be satisfied by $p$ are the boundary condition (39), mass balances (1), (13), (21) and constitutive equations (26) and (27).

A general solution for these equations can be sought for by assimilating the cracks to a distribution of point sources or sinks in $\Omega$. The pressure field solution for a point source in steady-state flow is $p(\underline{x})=f\|\underline{x}\|^{-1}$ where $f$ is a constant related to the source intensity. This solution is based on the mathematical identity:

$$
\Delta\|\underline{x}\|^{-1}=-4 \pi \delta(\underline{x})
$$

where $\delta$ represents the Dirac distribution. By using this result, a solution for $p(\underline{x})$ in $\Omega$ can be a priori sought for in the following form:

$$
p(\underline{x})=p_{\infty}(\underline{x})+\sum_{j} \int_{\Gamma_{j}} \frac{f^{j}(\underline{s})}{\left\|\underline{x}-\underline{z}^{j}(\underline{s})\right\|} d s+\sum_{i} \int_{L^{i}} \frac{b^{i}(l)}{\left\|\underline{x}-\underline{z}^{i}(l)\right\|} d l
$$

where $f^{j}$ and $b^{i}$ are continuous and integrable scalar functions respectively on $\Gamma_{j}$ and $L^{i}$. Such a solution satisfies automatically the infinite boundary conditions and mass balance $\Delta p=0$ in 
the matrix $\Omega-\Gamma$. The question in the following is to determine the conditions that make (41) fulfil the mass balance at every point on $\Gamma$.

For a point $\underline{z}$ on $\Gamma_{j}-L$ with $\underline{z}=\underline{z}^{j}(\underline{s})$, the mass balance (21) is reduced to:

$$
\llbracket \underline{v}(\underline{z}) \rrbracket \cdot \underline{n}(\underline{s})=-\nabla_{s} \cdot \underline{q}(\underline{s})
$$

where $\underline{n}(\underline{s})$ is the unit normal to $\Gamma_{j}$ at $\underline{z}$. The contributions of $p_{\infty}$ and of the integrals on $\Gamma_{j}$ ' with $j^{\prime} \neq j$ to the velocity field $\underline{v}(\underline{x})=-k \nabla p(\underline{x})$ remain continuous across $\Gamma_{j}$. The discontinuity $\llbracket \underline{v}(\underline{z}) \rrbracket$ on this surface results only from the integral on $\Gamma_{j}$, and is given by $\llbracket \underline{v}(\underline{z}) \rrbracket=-k \llbracket \nabla p^{j}(\underline{z}) \rrbracket$ where:

$$
p^{j}(\underline{x})=\int_{\Gamma_{j}} \frac{f^{j}(\underline{s})}{\left\|\underline{x}-\underline{z}^{j}(\underline{s})\right\|} d s
$$

It has been established in Appendix $C$ that for a pressure field having the expression (43), and for $\underline{x}=\underline{z}(\underline{s})$ on $\Gamma_{j}, \llbracket \nabla p^{j}(\underline{x}) \rrbracket \cdot \underline{n}(\underline{s})=-4 \pi f^{j}(\underline{s})$. Then, the mass balance (42) will be satisfied if we take:

$$
f^{j}(\underline{s})=\frac{-1}{4 \pi k} \nabla_{s} \cdot \underline{q}(\underline{s})
$$

Replacing by this expression in the surface integral term in (41) and then integrating by parts, one finds:

$$
\int_{\Gamma_{j}} \frac{\nabla}{\| \underline{x}_{s} \cdot \underline{q}^{j}} d s=\int_{\partial \Gamma_{j}} \frac{\underline{q}^{j}(\underline{s}) \cdot \underline{m}^{j}(\underline{s})}{\left\|\underline{x}-\underline{z}^{j}(\underline{s})\right\|} d l-\int_{\Gamma_{j}} \frac{\underline{q}^{j}(\underline{s}) \cdot\left(\underline{x}-\underline{z}^{j}(\underline{s})\right)}{\left\|\underline{x}-\underline{z}^{j}(\underline{s})\right\|^{3}} d s
$$

Replacing by (45) in (41) a sum on all the crack boundaries $\partial \Gamma_{j}$ is found. The boundary $\partial \Gamma_{j}$ is constituted of intersections of $\Gamma_{j}$ with other cracks on the lines $L^{i}$, designated by $\gamma_{j}^{i}$. The lines $L^{i}$ here include also the border of cracks ending in the body with only one value for $j$. There is no intersection of $\Gamma_{j}$ with $\partial \Omega$ since the cracks have finite size and the domain $\Omega$ is infinite. When summing on all the lines $\gamma_{j}^{i}$ for different $\partial \Gamma_{j}$, by inverting the summation order between $i$ and $j$, an integral on the lines $L^{i}$ is found:

$$
\sum_{j} \int_{\partial \Gamma_{j}} \frac{\underline{q}^{j}(\underline{s}) \cdot \underline{m}^{j}(\underline{s})}{\left\|\underline{x}-\underline{z}^{j}(\underline{s})\right\|} d l=\sum_{j} \sum_{i} \int_{\gamma_{j}^{i}} \frac{\underline{q}^{j}(l) \cdot \underline{m}^{j}(l)}{\left\|\underline{x}-\underline{z}^{j}(l)\right\|} d l=\sum_{i} \int_{L^{i}} \sum_{j} \frac{q^{j}(l) \cdot \underline{m}^{j}(l)}{\left\|\underline{x}-\underline{z}^{j}(l)\right\|} d l
$$

$q^{j}(l)$ represents the infiltration computed on the surface $\Gamma_{j}$ at the point on $\gamma_{j}^{i}$ with curvilinear abscise $l$. On the last integral, on a given line $L^{i}$ and at given curvilinear abscise $l$ on this line, all the $\underline{z}^{j}(l)$ for different values of $j$ take a unique value $\underline{z}^{i}(l)$. Then, we can write:

$$
\sum_{i} \int_{L^{i}} \sum_{j} \frac{\underline{q}^{j}(l) \cdot \underline{m}^{j}(l)}{\left\|\underline{x}-\underline{z}^{j}(l)\right\|} d l=\sum_{i} \int_{L^{i}} \frac{\sum_{j} \underline{q}^{j}(l) \cdot \underline{m}^{j}(l)}{\left\|\underline{x}-\underline{z}^{i}(l)\right\|} d l
$$


Replacing by these relations in (41) results in:

$$
p(\underline{x})=p_{\infty}(\underline{x})+\frac{1}{4 \pi k} \sum_{j} \int_{\Gamma_{j}} \frac{\underline{q}^{j}(\underline{s}) \cdot\left(\underline{x}-\underline{z}^{j}(\underline{s})\right)}{\left\|\underline{x}-\underline{z}^{j}(\underline{s})\right\|^{3}} d s+\frac{1}{4 \pi k} \sum_{i} \int_{L^{i}} \frac{B^{i}(l)}{\left\|\underline{x}-\underline{z}^{i}(l)\right\|} d l
$$

with:

$$
B^{i}(l)=4 \pi k b^{i}(l)-\sum_{j} \underline{q}^{j}(l) \cdot \underline{m}^{j}(l)
$$

Let us now examine the nature of the singularity of the right-side of (48) at a point $\underline{x}$ on the line $L^{i}$. First, a small interval on this line with length $2 r$ and centred on $\underline{x}$ can be considered and parameterized by $l \in[-r, r]$. The contribution of the line integral to this singularity can be determined by making a first order expansion of different functions with respect to $l$ in the neighbourhood of $\underline{x}$. It yields $\left\|\underline{x}-\underline{z}^{j}(l)\right\| \approx l, \quad \underline{q}^{j}(l) \approx \underline{q}^{j}(\underline{x})=\underline{q}^{j}, \underline{m}^{j}(l) \approx \underline{m}^{j}(\underline{x})=\underline{m}^{j}$, $b^{i}(l) \approx b^{i}(\underline{x})$, and then :

$$
\int_{-r}^{r} \frac{B^{i}(l)}{\left\|\underline{x}-\underline{z}^{i}(l)\right\|} d l \approx 2\left(4 \pi k b^{i}(\underline{x})-\sum_{j} \underline{q}^{j} \cdot \underline{m}^{j}\right) \int_{0}^{r} \frac{d l}{l}
$$

The contribution of the surface integral on $\Gamma_{j}$ in the right-side of (48) to the singularity is deduced from integration on a half-circle $C_{h}$ with radius $r$ and originated at $\underline{x}$ (Figure 6). We parameterize this half-circle with the polar coordinates $(l, \theta)$ where $l \in[0, r]$ and $\theta \in[0, \pi]$. For a point $\underline{z}(\underline{s})$ in the neighbourhood of $\underline{x}$ in $\Gamma_{j}$, a first order expansion of $\underline{q}(\underline{s}) \cdot\left[\underline{z}^{j}(\underline{s})-\underline{x}\right]$ with respect to $l$ yields $\underline{z}-\underline{x} \approx l \cos \theta \underline{t}-l \sin \theta \underline{m}^{j}$ and $\underline{q}(\underline{s}) \approx \underline{q}^{j}(\underline{x})=\underline{q}^{j}$. Hence:

$$
\int_{C_{h}} \frac{\underline{q}(\underline{s})^{j} \cdot\left(\underline{x}-\underline{z}^{j}(\underline{s})\right)}{\left\|\underline{x}-\underline{z}^{j}(\underline{s})\right\|^{3}} d s \approx \int_{0}^{r} \int_{0}^{\pi} \frac{-\left(\underline{q}^{j} \cdot \underline{t}\right) \cos \theta+\left(\underline{q}^{j} \cdot \underline{m}^{j}\right) \sin \theta}{l} d \theta d l=2\left(\underline{q}^{j} \cdot \underline{m}^{j}\right) \int_{0}^{r} \frac{d l}{l}
$$

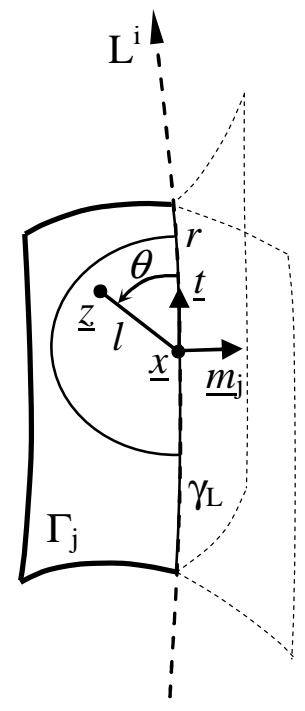

Figure 6: Determination of the singularity in the neighbourhood of a point $\underline{x}$ on an intersection line 
Comparing (51) to (50), it is found that the singularity due to the surface integral compensates exactly the part $-2 \sum_{j} \underline{q}^{j} \cdot \underline{m}^{j}$ coming from the line integral. This results from the fact that the surface integral (41) is not singular if the function $f$ is integrable. Therefore, the singularity coming from the line integral in (41) is not compensated. So, to have a finite pressure $p$ at $\underline{x \in} L^{i}$ requires taking $b^{i}(\underline{x})=0$. With this result, the potential solution is written as (48) in which $B^{i}(l)=-\sum_{j} \underline{q}^{j}(l) \cdot \underline{m}^{j}(l)$.

The mass balance (21) on intersection lines has not yet been taken into account. By introducing this condition, we find $B^{i}=0$ and the following expression is finally obtained for the potential solution:

$$
p(\underline{x})=p_{\infty}(\underline{x})+\frac{1}{4 \pi k} \sum_{j} \int_{\Gamma_{j}} \frac{\underline{q}^{j}(\underline{s}) \cdot\left(\underline{x}-\underline{z}^{j}(\underline{s})\right)}{\left\|\underline{x}-\underline{z}^{j}(\underline{s})\right\|^{3}} d s
$$

This expression gives the solution of pressure field in terms of the unknown field $\underline{q}^{j}$ on crack surfaces.

The expression (52) satisfies obviously boundary condition (39) and mass conservation (1). It would be possible to begin directly with this expression as a potential solution and to show that it satisfies well mass balances (21) and (13). But demonstration of these properties in this case would be much more difficult since the singularity of the integrand in (52) has a greater order than in (41).

The potential solution here means that, for arbitrary $q^{j}$ fields, the expression (52) satisfies all equations of the flow problem except for the constitutive flow law in crack surfaces. Introducing the relation (27) in (52), a system of singular integral equations on the pressure field is obtained that allows computing this field. This system will be presented in the next section for the general case of anisotropic matrix.

\section{Extension to anisotropic matrix}

In this section, the linear transformation (29) is used to extend the solution (52) to anisotropic matrices. Let the infinite body $\Omega$ with uniform matrix permeability $\boldsymbol{k}$ contain a family of cracks $\Gamma_{j}$ and be submitted at its infinite boundary to the pressure conditions $p_{\infty}$. Provided that $\boldsymbol{k}$ is symmetric and positive-definite, we designate by $\boldsymbol{M}=\sqrt{\boldsymbol{k}^{-1}}$ the positive and symmetric matrix that satisfies $\boldsymbol{M} \boldsymbol{M}=\boldsymbol{k}^{-1}$. We take $\zeta=1$. The transformed problem corresponds to an infinite body with isotropic permeability $\tilde{\boldsymbol{k}}=\boldsymbol{\delta}$. For this transformed problem, the solution (52) reads:

$$
\tilde{p}(\underline{\tilde{x}})=\tilde{p}_{\infty}(\underline{\tilde{x}})+\frac{1}{4 \pi} \sum_{j} \int_{\tilde{\Gamma}_{j}} \frac{\underline{\tilde{q}}^{j}(\underline{s}) \cdot\left(\underline{\tilde{x}}-\underline{\tilde{z}}^{j}(\underline{s})\right)}{\left\|\underline{\tilde{x}}-\tilde{z}^{j}(\underline{s})\right\|^{3}} d \tilde{s}
$$


As noticed in Appendix A, it is not necessary to change the parameterization $\underline{s}$ for the transformed surfaces $\tilde{\Gamma}_{j}$. Only the functions $\underline{\tilde{z}}^{j}(\underline{s})$ and also the surface element $d \tilde{s}$ are changed in the geometrical transformation. The expressions of all transformed variables $\left(\underline{\tilde{x}}, \tilde{z}, \tilde{p}, \tilde{p}_{\infty}, \underline{\tilde{q}}, d \tilde{s}\right)$ as functions of initial variables $\left(\underline{x}, \underline{z}, p, p_{\infty}, \underline{q} \underline{d s}\right)$ are given in Section 3 or in Appendix A. Substituting by these expressions in (53), the final expression of the potential solution for flow in an anisotropic matrix is obtained as follows:

$$
p(\underline{x})=p_{\infty}(\underline{x})+\frac{1}{4 \pi \sqrt{|\boldsymbol{k}|}} \sum_{j} \int_{\Gamma_{j}} \frac{\underline{q}^{j}(\underline{s}) \cdot \boldsymbol{k}^{-1} \cdot\left(\underline{x}-\underline{z}^{j}(\underline{s})\right)}{\left[\left(\underline{x}-\underline{z}^{j}(\underline{s})\right) \cdot \boldsymbol{k}^{-1} \cdot\left(\underline{x}-\underline{z}^{j}(\underline{s})\right)\right]^{3 / 2}} d s
$$

It is worth noting that the only significant assumption to derive this solution was that the matrix permeability $\boldsymbol{k}$ is uniform: the crack surfaces can be curved and have arbitrary multiple intersections. Moreover, the constitutive flow model in fractures is not specified and can contain arbitrary non linearities.

By using the tangential flow law, $q$ in (54) can be replaced by its expression function of the pressure. Then if $\underline{x}$ scans the fracture surfaces, a system of equations is obtained that involves only the pressure on the fractures. The resolution of this system allows determining, first, the pressures in the fractures, then, the infiltration $q$ by using the tangential flow law, and finally, by returning back to (54) and allowing $\underline{x}$ to scan the whole space, the pressure field in the whole body. Ultimately, in this way, the initial 3-D flow problem is reduced to resolution of a system of equations involving only pressures on fractures surfaces, i.e., to a 2-D type problem.

For instance, in the case of a linear tangential flow law in fractures, $\underline{q}(\underline{s})=-c(\underline{s}) . \nabla_{s} p(\underline{s})$, the following system of singular integral equations on the pressure field is obtained:

$$
p(\underline{x})=p_{\infty}(\underline{x})-\frac{1}{4 \pi \sqrt{|\boldsymbol{k}|}} \sum_{j} \int_{\Gamma_{j}} \frac{\nabla_{s}^{j} p(\underline{s}) \cdot\left[\boldsymbol{c}^{j}(\underline{s}) \boldsymbol{k}^{-1}\right] \cdot\left(\underline{x}-z^{j}(\underline{s})\right)}{\left[\left(\underline{x}-z^{j}(\underline{s})\right) \boldsymbol{k}^{-1} \cdot\left(\underline{x}-z^{j}(\underline{s})\right)\right]^{3 / 2}} d s
$$

The anisotropic conductivity $\boldsymbol{c}^{j}$ can vary along the crack surface. This equation can be numerically solved for instance by using a set of collocation points [26]. Exactly the same equations (54) and (55) were obtained in the two-dimensional case [14], with the difference that in the right-side of these equations, the factor $4 \pi$ in the denominator is replaced by $2 \pi$ and the power $3 / 2$ by 2 . The solution in two-dimensional case was used to elaborate numerical methods that proved their efficiency [27]. We have used (55), in an undergoing work, to determine closed-form solution for flow in disc-shaped cracks in an infinite matrix submitted to a far-filed gradient, and apply the result to effective permeability calculation. The elaboration of the numerical method for three-dimensional case requires some specific investigation that we hope to present in a future paper. 


\section{Conclusions and perspectives}

A complete mathematical formulation for the flow problem in a fractured porous body is presented in this paper which fully integrates mass balance conditions along fracture intersection lines. A main result is that mass exchanges on intersection lines between fractures are independent from the exchanges with the matrix. They have the same expressions as for a fracture network in an impermeable matrix. As a consequence, on a crack tip, where a crack ends in the matrix, the normal component of the infiltration to the crack tip line is null. These results, that extend those found for 2D case in previous works, allow significant simplifications for mathematical and numerical modelling of the flow problem.

A potential solution for the steady state flow in an infinite fractured body is presented based on singular integral equations. This solution allows to determine the flow (pressure and velocity) field in a 3-D fractured porous body by solving equations involving only the pressures on the fracture surfaces and, thus, to reduce the 3-D problem in a 2-D problem. The numerical resolution is then much simplified because it requires only discretization of fracture surfaces.

An interesting case to be studied by using the solution (55) is that of a single disc-shaped crack in an infinite matrix under uniform farfield pressure gradient. A closed-form or a semianalytical solution for this case can be found which opens the way for many theoretical studies, specially related to the effective permeability of microcracked porous materials. Also, the potential solution (55) that supposes an infinite body, can be extended to finite domains by using classical results of Boundary Element methods [26]. These extensions will be addressed in a future paper.

Finally, a main assumption in this paper has been that the matrix and fractures are saturated and so no capillary phenomena are to be taken into account. In this case the thermodynamic considerations for flow in porous media $[28,29]$ reduce to mass balance conditions. However, some interesting physical and industrial applications, like flow in petroleum reservoirs and $\mathrm{CO}_{2}$ storage problems, imply multiphase flow in cracked porous media. In this case, the effects of capillary forces in matrix and fractures and the related thermodynamic questions have to be carefully analysed and fully taken into account. We hope to integrate these questions in a further extension of the present work to unsaturated cracked porous materials. 


\section{Appendix A: Curved surfaces and geometrical transformation}

\section{Curved surface}

The curved surface $\Gamma$ is defined by a smooth function $\underline{z}(\underline{s})$ from $\mathbb{R}^{2}$ to $\mathbb{R}^{3}$. The parameter $\underline{s}=\left(s_{1}, s_{2}\right)$ varies in a domain of $\mathbb{R}^{2}$. The natural (not necessarily orthonormal) basis tangent to $\Gamma$ at a point $\underline{x}(\underline{s})$ is given by $\left(\underline{t}_{1}, \underline{t}_{2}\right)$ with $\underline{t} \alpha=\partial \underline{x} / \partial s_{\alpha}(\alpha=1,2)$. The unit normal is given as $\underline{n}=\left(\underline{t}_{1} \times \underline{t}_{2}\right) /\left\|\underline{t}_{1} \times \underline{t}_{2}\right\|$. The surface element $d s=\left\|\underline{t}_{1} \times \underline{t}_{2}\right\| d s_{1} d s_{2}$ is also equal to $d s=\left|\underline{t}_{1}, \underline{t}_{2}, \underline{n}\right| d s_{1} d s_{2}$ where $\left|\underline{t}_{1}, \underline{t}_{2}, \underline{n}\right|$ is the determinant of the matrix having for columns the vectors $\left(\underline{t}_{1}, \underline{t}_{2}, \underline{n}\right)$. For a curve $L$ inscribed on $\Gamma$, the Darboux frame $(\underline{t}, \underline{n}, \underline{m})$ at a point $\underline{x}$ on $L$ is constituted of the unit tangent $\underline{t}$ to $L$, the unit normal $\underline{n}$ to $\Gamma$ and the unit tangent normal $\underline{m}=$ $\underline{t} \times \underline{n}$ that is tangent to $\Gamma$ and normal to $L$ (Figure 7 ).

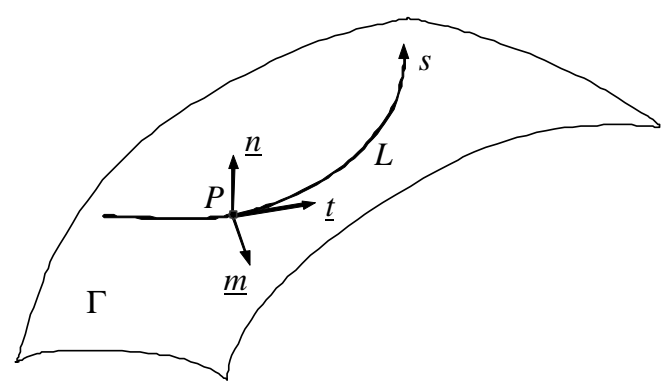

Figure 7: Darboux frame for a line $L$ inscribed on a curve surface $\Gamma$

The Christoffel symbols $C_{\alpha \beta}^{\gamma}$ and $b_{\alpha \beta}$ are defined by the relations:

$$
\partial \underline{t}_{\beta} / \partial s_{\alpha}=C_{\alpha \beta}^{\gamma} \underline{t}_{\gamma}+b_{\alpha \beta} \underline{n}
$$

The relation $\underline{n} \cdot \underline{t}_{\alpha}=0$ yields :

$$
\left(\partial \underline{n} / \partial s^{\beta}\right) \cdot \underline{t}_{\alpha}=-\underline{n} . \partial \underline{t}_{\alpha} / \partial s^{\beta}=b_{\alpha \beta}
$$

\section{Geometrical transformation}

A geometrical transformation $\underline{\tilde{x}}=\boldsymbol{M} . \underline{x}$ changes $\Gamma$ to a surface $\tilde{\Gamma}$. The same parameterization $\underline{s}$ can be maintained for $\tilde{\Gamma}$, but the function $\underline{z}(\underline{s})$ is changed into the function $\underline{\tilde{z}}(\underline{s})=\boldsymbol{M} \cdot \underline{z}(\underline{s})$. The natural tangent frame on $\tilde{\Gamma}$ is given by $\underline{T}_{\alpha}=\partial \tilde{x} / \partial s_{\alpha}=M \cdot t_{\alpha}(\alpha=1,2)$.

$\underline{\text { Unit normal: }}$ The unit normal $\underline{\tilde{n}}$ on the surface $\tilde{\Gamma}$ at the point $\underline{\tilde{x}}$ is related to the unit normal $\underline{n}$ on $\Gamma$ at $\underline{x}$ by $\underline{\tilde{n}}=h_{n} \boldsymbol{M}^{-1, \mathrm{~T}} \cdot \underline{n}$, where $h_{n}=\left\|\boldsymbol{M}^{-1, \mathrm{~T}} \cdot \underline{n}\right\|^{-1}$. This results from the fact that if $\delta \underline{x}$ is tangent to $\Gamma$ then $\delta \underline{\tilde{x}}=M . \delta \underline{x}$ is tangent to $\tilde{\Gamma}$ and $\underline{n}$ and $\underline{\tilde{n}}$ have to satisfy respectively $\underline{n} . \delta \underline{x}=0$ and $\underline{\tilde{n}} \cdot \delta \underline{\tilde{x}}=0$.

Darboux frame: A curve $L$ on the surface $\Gamma$ is transformed to $\tilde{L}$ on $\tilde{\Gamma}$. The Darboux frame $(\underline{\tilde{t}}, \underline{\tilde{n}}, \underline{\tilde{m}})$ of $\tilde{L}$ at the point $\underline{\tilde{x}}$ is related to $(\underline{t}, \underline{n}, \underline{m})$ at $\underline{x}$ by $\underline{\tilde{t}}=h_{\mathrm{t}} \boldsymbol{M} \cdot \underline{t}$ with $h_{t}=\|\boldsymbol{M} \cdot \underline{t}\|^{-1}$, $\underline{\tilde{n}}$ already defined and $\underline{\tilde{m}}=\underline{\tilde{t}} \times \underline{\tilde{n}}$.

Surface element: The surface element on $\tilde{\Gamma}$ is $d \tilde{s}=\left|\underline{T}_{1}, \underline{T}_{2}, \tilde{n}\right| d s_{1} d s_{2}$ where the natural tangent basis $\left\{\underline{T}_{\alpha}\right\}$ is given by $\underline{T}_{\alpha}=\partial \tilde{x} / \partial s_{\alpha}=M \cdot t_{\alpha}$. By using the mathematical property: 


$$
\left|\boldsymbol{M} \cdot \underline{a}_{1}, \boldsymbol{M} \cdot \underline{a}_{2}, \boldsymbol{M} \cdot \underline{a}_{3}\right|=|\boldsymbol{M}|\left|a_{1}, \underline{a}_{2}, \underline{a}_{3}\right|
$$

we compute:

$$
\left|\underline{T}_{1}, \underline{T}_{2}, \underline{\tilde{n}}\right|=h_{n}|\boldsymbol{M}|\left|\underline{t}_{1}, \underline{t}_{2}, \boldsymbol{N} . \underline{n}\right|
$$

with:

$$
\boldsymbol{N}=\boldsymbol{M}^{-1} \boldsymbol{M}^{-1, \mathrm{~T}}
$$

The vector $N . \underline{n}$ can be decomposed in its components parallel and normal to $\underline{n}: \quad N . \underline{n}=\lambda \underline{n}+$ $\underline{T}$ with $\underline{n} . \underline{T}=0$ and $\lambda=\underline{n} . N . \underline{n}$. Beside, we can decompose $\left|\underline{t}_{1}, \underline{t}_{2},(\lambda \underline{n}+\underline{T})\right|=\lambda\left|\underline{t}_{1}, \underline{t}_{2}, \underline{n}\right|+\mid \underline{t}_{1}, \underline{t}_{2}$, $\underline{T} \mid$ where the last determinant vanishes since $\left(\underline{t}_{1}, \underline{t}_{2}, \underline{T}\right)$ are coplanar. Replacing by this in (A.4) and using:

$$
\underline{n} . N \cdot \underline{n}=h_{n}^{-2}
$$

we find: $\left|\underline{T}_{1}, \underline{T}_{2}, \underline{\tilde{n}}\right|=h_{n}^{-1}|\boldsymbol{M}|\left|\underline{t}_{1}, \underline{t}_{2}, \underline{n}\right|$ and then $d \tilde{s}=h_{n}^{-1}|\boldsymbol{M}| d s$.

Christoffel symbols : The variation of the natural basis $\left\{\underline{T}_{\alpha}\right\}$ on $\tilde{\Gamma}$ is given by: $\partial \underline{T}_{\beta} / \partial s_{\alpha}=M . \partial \underline{t}_{\beta} / \partial s_{\alpha}=C_{\alpha \beta}^{\gamma} \underline{T}_{\gamma}+b_{\alpha \beta} M . \underline{n}$. Then if :

$$
M . \underline{n}=\tau^{\gamma} \underline{T}_{\gamma}+v \underline{\tilde{n}}
$$

we find: $\partial \underline{T}_{\beta} / \partial s_{\alpha}=\tilde{C}_{\alpha \beta}^{\gamma} \underline{T}_{\gamma}+\tilde{b}_{\alpha \beta} \underline{\tilde{n}}$ with:

$$
\tilde{C}_{\alpha \beta}^{\gamma}=C_{\alpha \beta}^{\gamma}+\tau^{\gamma} b_{\alpha \beta}, \quad \tilde{b}_{\alpha \beta}=v b_{\alpha \beta}
$$

Also, from (A.7) it can be deduced that $\underline{n}=\tau^{\gamma} \underline{t}_{\gamma}+v h_{n} N . \underline{n}$, and this, with (A1.6), yield $\mathrm{v}=h_{n}$, and:

$$
N . \underline{n}=h_{n}^{-2}\left(\underline{n}-\tau^{\gamma} \underline{t}_{\gamma}\right)
$$

Derivation of (A.6) with respect to $s^{\alpha}$ yields $\partial h_{n} / \partial s^{\alpha}=-h_{n}{ }^{3} \underline{n} . N .\left(\partial \underline{n} / \partial s^{\alpha}\right)$. Using (A.6), (A.2) and the property $\underline{n}$. $\partial \underline{n} / \partial s^{\alpha}=0$ that results from $\underline{n} \cdot \underline{n}=1$, it can be established that $\underline{n} \cdot N .\left(\partial \underline{n} / \partial s^{\alpha}\right)$ $=h_{n}^{-2} \tau^{\gamma} b_{\alpha \gamma}$ and then:

$$
\partial h_{\mathrm{n}} / \partial s^{\alpha}=-h_{n} \tau^{\beta} b_{\alpha \beta}
$$

\section{Appendix B: Transformation of infiltration type vector fields}

A tangent vector field $\underline{u}$ through $\Gamma$ is called infiltration type field when it is transformed as:

$$
\underline{\tilde{u}}(\underline{\tilde{z}})=h_{n}(\underline{z}) \boldsymbol{M} \cdot \underline{u}(\underline{z})
$$

For these fields then $\underline{\tilde{u}}=\tilde{u}^{\alpha} \underline{T}_{\alpha}$ with $\tilde{u}^{\alpha}=h_{n} u^{\alpha}$.

Surface divergence: The surface divergence of $\underline{\tilde{u}}$ is transformed as follows:

$$
\begin{aligned}
\tilde{\nabla}_{s} \cdot \underline{\tilde{u}}=\partial \tilde{u}^{\alpha} / \partial s^{\alpha}+\tilde{u}^{\beta} \tilde{C}_{\alpha \beta}^{\alpha} & =h_{n} \partial u^{\alpha} / \partial s^{\alpha}+u^{\alpha} \partial h_{n} / \partial s^{\alpha}+h_{n} u^{\beta}\left(C_{\alpha \beta}^{\alpha}+\tau^{\alpha} b_{\alpha \beta}\right) \\
& =h_{n} \nabla_{s} \cdot \underline{u}+u^{\beta}\left(\partial h_{n} / \partial s^{\beta}+h_{n} \tau^{\alpha} b_{\alpha \beta}\right)
\end{aligned}
$$

Replacing by (A.9) in (B.2) the following result is found:

$$
\tilde{\nabla}{ }_{s} \cdot \underline{\tilde{u}}=h_{n} \nabla_{s} \cdot \underline{u}
$$

Normal infiltration on a boundary line: The normal component of $\underline{u}$ on a boundary line $L$ inscribed on $\Gamma$ is given by $\underline{u} . \underline{m}$ where $\underline{m}$ is the tangent normal of $L$ on $\Gamma$ (see Darboux frame). 
Using $\underline{m}=\underline{t} \times \underline{n}$ the scalar product is transformed in mixed product: $\underline{u} \cdot \underline{m}=|\underline{u}, \underline{t}, \underline{n}|$. The transformation rules for $\underline{\tilde{u}}, \underline{\tilde{t}}, \underline{\tilde{n}}$ and the relations (A.3) and (A.5) then yield:

$\underline{\tilde{u}} . \underline{\tilde{m}}=|\underline{\tilde{u}}, \underline{\tilde{t}}, \underline{\tilde{n}}|=h_{t}\left(h_{n}\right)^{2}\left|\boldsymbol{M} \underline{u}, \boldsymbol{M} \cdot \underline{t}, \boldsymbol{M}^{-1, \mathrm{~T}} \cdot \underline{n}\right|=h_{t}\left(h_{n}\right)^{2}|\boldsymbol{M}||\underline{u}, \underline{t}, \boldsymbol{N} . \underline{n}|$

Decomposition (A.9) of $N . \underline{n}$ with the same techniques used here above for the surface element $d s$ allows demonstrating that: $|\underline{u}, \underline{t}, N \cdot \underline{n}|=\left(h_{n}\right)^{-2}|\underline{u}, \underline{t}, \underline{n}|$ and then:

$$
\underline{\tilde{u}} \cdot \underline{\tilde{m}}=h_{t}|\boldsymbol{M}| \underline{u} \cdot \underline{m}
$$

\section{Appendix C: Velocity discontinuity and divergence on the crack surface}

Consider a smooth surface $\Gamma$ in $\mathbb{R}^{3}$ defined by the function $\underline{z}(\underline{s})$ where $\underline{s}=\left(s_{1}, s_{2}\right)$, and the function $p(\underline{x})$ defined in $\Omega \subseteq \mathbb{R}^{3}$ by:

$$
p(\underline{x})=\int_{\Gamma} \frac{f(\underline{s})}{\|\underline{z}(\underline{s})-\underline{x}\|} d s
$$

where $f$ is a continuous and integrable scalar function defined on $\Gamma$, and $d s$ is the surface element on $\Gamma$. The pressure gradient is computed as follows:

$$
\nabla p(\underline{x})=\int_{\Gamma} f(\underline{s}) \underline{J}(\underline{x}, \underline{s}) d s ; \quad \underline{J}(\underline{x}, \underline{s})=\frac{\underline{z}(\underline{s})-\underline{x}}{\|\underline{z}(\underline{s})-\underline{x}\|^{3}}
$$

The gradient $\nabla p(\underline{x})$ is discontinuous at a point $\underline{x}=\underline{z}_{0}=\underline{z}(0)$ on $\Gamma$. The jump $\llbracket \nabla p(\underline{x}) \rrbracket \cdot \underline{n}_{0}$ across $\Gamma$ where $\underline{n}_{0}$ is unit normal to $\Gamma$ at the point $\underline{x}$ is defined by:

$$
\llbracket \nabla p(\underline{x}) \rrbracket \cdot \underline{n}_{0}=\lim _{\gamma \rightarrow 0^{+}}\left(\nabla p\left(\underline{x}+\gamma \underline{n}_{0}\right)-\nabla p(\underline{x}-\gamma \underline{n})\right) \cdot \underline{n}_{0}
$$

For $\gamma=0$, the integrand $\underline{J}\left(\left(\underline{x}+\underline{\gamma}_{0}\right), \underline{s}\right)$ is singular at $\underline{s}=0$ and this causes the difference of the $\lim \underline{\nabla} p\left(\underline{z}_{0}+\gamma \underline{n}_{0}\right) . \underline{n}_{0}$ for $\gamma \rightarrow 0^{+}$and $\gamma \rightarrow 0^{-}$. We can limit the integration on a small zone around $\underline{x}$ on $\Gamma$ since outside this disc $\underline{J}((\underline{x}+\underline{\gamma} \underline{n}), \underline{s})$ is non singular for $\gamma \rightarrow 0$, and does not contribute to the jump. We assume that the local parameterization $\underline{z}\left(s_{1}, s_{2}\right)$ for $\Gamma$ is defined in such a way that $\underline{x}$ $=\underline{z}(0,0)$ and $\left(s_{1}, s_{2}\right)$ define locally a system of Cartesian coordinates, i.e., $\underline{t}_{1}$ and $\underline{t}_{2}$ defined by $\underline{t}_{\alpha}=\partial \underline{z} / \partial \mathrm{s}_{\alpha}(\alpha=1,2)$ are two perpendicular unit vectors. The small zone considered around $\underline{x}$ is the image $D$ of the disc of radius $r$ in the space $\left(s_{1}, s_{2}\right)$ centred at $\underline{s}=(0,0)$. To compute the integral on this small zone, we can replace all the functions by they first order expansion in $\left(s_{1}, s_{2}\right)$ :

$$
\begin{aligned}
& \underline{z}(\underline{s})=\underline{z}_{0}+s_{\alpha} \underline{t}_{\alpha}+\underline{\mathrm{o}}_{1}(\underline{s}) \\
& f(\underline{s})=f_{0}+f_{\alpha S_{\alpha}}+\mathrm{o}_{2}(\underline{s})
\end{aligned}
$$

where $f_{\alpha}=\partial f / \partial s_{\alpha}$ at the point $\underline{s}=(0,0)$, and $\lim _{\underline{s} \rightarrow 0} \underline{o}_{1}(\underline{s})=0, \lim _{\underline{s} \rightarrow 0} o_{2}(\underline{s})=0$.

By choosing polar coordinates $(r, \theta)$ in the disc $D$, i.e, $s_{1}=r \cos \theta, s_{2}=r \sin \theta$, and reducing the integrand to its first order expansion, the following expression is obtained: 


$$
\begin{aligned}
\underline{n}_{0} \cdot \int_{D} f(\underline{s}) \underline{J}\left(\underline{z}_{0}+\gamma \underline{n}_{0}, \underline{s}\right) d s & \approx-\gamma \int_{r=0}^{R} \int_{\theta=0}^{2 \pi} \frac{f_{0}+f_{1} r \cos \theta+f_{2} r \sin \theta}{\left(\gamma^{2}+r^{2}\right)^{3 / 2}} r d r d \theta \\
& =-2 \pi f_{0} \gamma\left(\frac{1}{|\gamma|}-\frac{1}{\sqrt{\gamma^{2}+R^{2}}}\right)
\end{aligned}
$$

It allows computing: $\lim _{\gamma \rightarrow 0^{+}} \nabla p\left(\underline{x}+\gamma \underline{n}_{0}\right)=-2 \pi f_{0}$ and $\lim _{\gamma \rightarrow 0^{-}} \underline{\nabla} p\left(\underline{x}+\gamma \underline{n}_{0}\right)=2 \pi f_{0}$. These results allow writing for a general point $\underline{x}=\underline{z}(\underline{s})$ on a crack surface $\Gamma$ :

$$
\llbracket \nabla p(\underline{z}(\underline{s})) \rrbracket \cdot \underline{n}(\underline{s})=-4 \pi f(\underline{s})
$$

\section{References}

[1] Blöcher M.G., Cacace M., Lewerenz B., Zimmermann G., Three dimensional modeling of fractured and faulted reservoirs: Framework and implementation. Chemie der Erde 70 (2010) S3, 145-153

[2] Castany, G.: Traité pratique des eaux souterraines. Editions Dunod, Paris (1967).

[3] Dershowitz, W. and Miller, I.: "Dual porosity fracture flow and transport," Geophysical Research Letters (1995) 22, No. 11, 1441.

[4] Bogdanov I.I., Mourzenko V.V. and Thovert J.F., Effective permeability of fractured porous media in steady state flow. Water Resources Research, Vol. 39, NO. 1, 1023, doi:10.1029/2001WR000756, 2003

[5] Granet S., Fabrie P., Lemonnier P., Quintard M., A two-phase f low simulation of a fractured reservoir using a new fissure element method, Journal of Petroleum Science and Engineering 32 (2001) 35- 52

[6] Mourzenko V.V, Bogdanov I.I., Thovert J.-F., Adler P.M., Three-dimensional numerical simulation of single-phase transient compressible flows and well-tests in fractured formations, Mathematics and Computers in Simulation (2010), doi:10.1016/j.matcom.2010.12.014

[7] Sarda S., Jeannin L., Basquet R., Bourbiaux B., Hydraulic Characterization of Fractured Reservoirs: Simulation on Discrete Fracture Models, SPE 77300, April 2002 SPE Reservoir Evaluation \& Engineering

[8] Lange A., Basquet R., Bourbiaux B., Hydraulic Characterization of Faults and Fractures Using a Dual Medium Discrete Fracture Network Simulator, SPE 88675, 2004.

[9] Arbogast, T., J. Douglas, and U. Hornung, Derivation of the double-porosity model of single phase flow via homogeneisation theory, SIAM, J. Math. Anal., 21, 823-836, 1990.

[10] Dormieux, L., Kondo, D.: Approche micromécanique du couplage perméabilité-endommagement, C.R. Mecanique 332, 135-140 (2004).

[11] Pouya A., Fouché O., "Permeability of 3D discontinuity networks: New tensors from boundaryconditioned homogenisation", Advances in Water Resources, 32 (2009) 303-314.

[12] Zimmerman, R.: Effective conductivity of a two-dimensional medium containing elliptical inhomogeneities. Proc. Roy. Soc. Ser. A 452(1950), 1713-1727 (1996)

[13] Liolios, P.A. Exadaktylos, G.E.: A solution of steady-state fluid flow in multiply fractured isotropic porous media, International Journal of Solids and Structures 43, 3960-3982 (2006). 
[14] Pouya, A., Ghabezloo S. 2010. Flow around a crack in a porous matrix and related problems. Transport in Porous Media 84(2): 511-532. [16-8] Lange A., Basquet R., Bourbiaux B., Hydraulic Characterization of Faults and Fractures Using a Dual Medium Discrete Fracture Network Simulator, SPE 88675, 2004.

[15] Barenblatt, G. I., Y. P. Zheltov, and I. N. Kochina, Basic concepts in the theory of seepage of homogeneous liquids in fissured rocks, Sov. Appl. Math. Mech., Engl. Transl., 24, 852-864, 1960.

[16] Adler, P. M., Porous Media: Geometry and Transports, Butterworth- Heinemann, Woburn, Mass., 1992.

[17] Aifantis, E. C., On Barrenblatt's problem, Int. J. Eng. Sci., 18, 857- 867, 1980.

[18] Wu Y.S., Pan L., Pruess K., A physically based approach for modelling multiphase fracture-matrix interaction in fractured porous media. Advances in Water Resources 27 (2004) 875-887.

[19] Simunek J., Jarvis N.J., van Genuchten M.Th., Gärdenäs A. "Review and comparison of models for describing non-equilibrium and preferential flow and transport in the vadose zone “, Journal of Hydrology 272 (2003) 14-35.

[20] Manzocchi T., Childs C., Walsh J.J.: Faults and fault properties in hydrocarbon flow models. Geofluids (2010), 10, 94-113.

[21] Guéguen, Y., Palciauskas V.: Introduction to Physics of Rocks, Princeton Univ. Press (1994).

[22] Gentier S.; Lamontagne E.; Archambault G., Riss J., 1997, “Anisotropy of flow in a fracture undergoing shear and its relationship to the direction of shearing and injection pressure, Int. J. RockMech. \&Min. Sci. 34:3-4, Paper No. 094.

[23] Barthélémy, J.-F.: Effective Permeability of Media with a Dense Network of Long and Micro Fractures. Transp Porous Med (2009) 76:153-178.

[24] Milton G.W.: The theory of composites, pp. 145-147, Cambridge University Press (2002).

[25] Pouya, A., Zaoui, A.: A transformation of elastic boundary value problems with application to anisotropic behavior, Int. Journal of Solids and Structures 43, 4937-4956 (2006).

[26] Bonnet M., "Equations intégrales et éléments de frontière”, CNRS Editions/Eyrolles, Paris (1995).

[27] Pouya, A., Vu, M. N., Modeling steady-state flow in fractured porous rocks by singular integral equations method. In Proceedings of the 13th International Conference of the International Association for Computer Methods and Advances in Geomechanics, Melbourne, 2011.

[28] Hassanizadeh, S. M., Gray, W. G., 1990. Mechanics and thermodynamics of multiphase flow in porousmedia including interphase boundaries, Advances in water resources, 13(4), 169-186.

[29] Hassanizadeh, S. M., Gray, W. G., 1993. Thermodynamics basis of capillary pressure in porous media. Water Resources Research. Vol 29, No 10, 3389-3405. 